\title{
Global and local aspects of the surface potential landscape for energy level alignment at organic-ZnO interfaces
}

\author{
Julia Stähler ${ }^{\mathrm{a}}$, Patrick Rinke ${ }^{\mathrm{b}}$ \\ ${ }^{a}$ Fritz-Haber-Institut der Max-Planck-Gesellschaft, Faradayweg 4-6, 14195 Berlin, \\ Germany \\ ${ }^{b}$ COMP/Department of Applied Physics, Aalto University, P.O. Box 11100, Aalto \\ FI-00076, Finland
}

\begin{abstract}
Hybrid systems of organic and inorganic semiconductors are a promising route for the development of novel opto-electronic and light-harvesting devices. A key ingredient for achieving a superior functionality by means of a hybrid system is the right relative position of energy levels at the interfaces of the two material classes. In this Perspective, we address the sensitivity of the potential energy landscape at various $\mathrm{ZnO}$ surfaces, a key ingredient for interfacial energy level alignment, by combining one- and two-photon photoelectron spectroscopy with density-functional theory calculations (DFT). We show that even very large work function changes $(>2.5 \mathrm{eV})$ do not necessarily have to be accompanied by surface band bending in $\mathrm{ZnO}$. Band bending - if it does occur - may be localized to few $\AA$ or extend over hundreds of nanometers with very different results for the surface work function and energy level alignment. Managing the delicate balance of different interface manipulation mechanisms in organic-inorganic hybrid systems will be a major challenge towards future applications.
\end{abstract}

Keywords: energy level alignment, zinc oxide, transparent conductive oxides, surface band bending, density-functional theory, ultrafast spectroscopy, inorganic/organic hybrid systems, electronic structure theory

\section{Introduction}

The increasing need for functional materials for optoelectronic and lightharvesting applications is a major motivation for research in physics and 
chemistry. In this context, numerous studies $[1,2,3]$ focus on the exploration of transparent conductive oxides - with their beneficial high charge carrier mobilities - and on organic materials exhibiting strong light-matter coupling $[4,5,6]$. When combined, these material classes promise hybrid functionality, that is, properties that go beyond those of the linear combination of the individual materials. Such superior functionality emerges from the distinct properties of the inorganic/organic (i/o) interface, be it a specific interfacial structure that modifies the optical and electronic properties or, simply, strong electronic or excitonic coupling across the interface. In both cases, detailed knowledge of the interface on microscopic length, time, and energy scales is required to achieve a profound and quantitative understanding of i/o hybrid systems. Ultimately, this could facilitate predictions for valuable, novel materials.

When a hybrid system is optically excited by the absorption of a photon, this excitation can relax through a multitude of pathways. If excitons are formed, they can transfer across or separate at the interface. The motion of excitons is governed by energy transfer and charge separation. While energy transfer is a key process in light-emitting devices and its rate is often limited by interfacial charge separation, charge separation is the essential mechanism in a solar cell. The balance between these processes defines the potential functionality of an interface. This balance depends strongly on the interfacial energy level alignment, i.e., the relative energetic position of frontier molecular orbitals with respect to the conduction and valence band $(\mathrm{CB}$ and $\mathrm{VB}$ ) of the inorganic semiconductor, the coupling of the $\mathrm{CB}$ and $\mathrm{VB}$ to the frontier orbitals, the band gaps, and exciton binding energies. As discussed in detail further below, knowledge and control over the potential energy landscape at such interfaces is, hence, vital for the implementation of novel hybrid materials for optoelectronic or ligh-harvesting applications.

A very promising and yet very challenging material for i/o hybrid systems is zinc oxide $(\mathrm{ZnO})$, which exhibits a wide band gap around $3.4 \mathrm{eV}$ [7], making it transparent to visible light. In this Perspective, we show how a quantitative and microscopic picture of the potential energy landscape at organic-ZnO interfaces can be reached by the combination of one- and twophoton photoemission experiments on high quality samples with densityfunctional theory (DFT) calculations. Our results demonstrate that it is important to not only reach a solid understanding of the global aspects of energy level alignment at i/o interfaces, but to also take local aspects into account. By local we here refer to all chemical or physical processes at or 


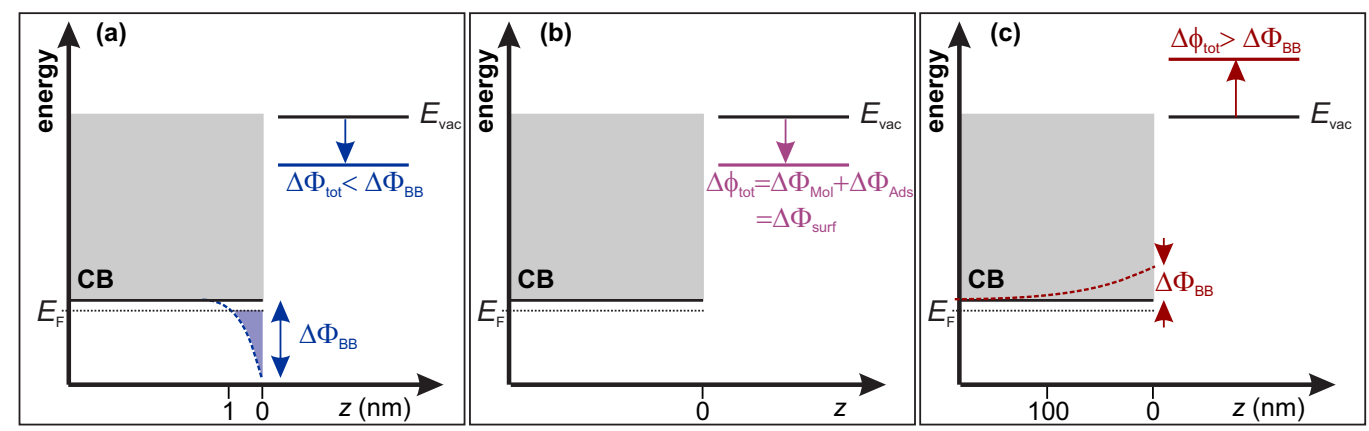

Figure 1: (color online) Three exemplary cases of ZnO surface electronic structure modifications. a) Charge transfer-induced downward surface band bending. The local character of the potential minima leads to a smaller work function reduction than expected from surface BB (cf. Section 5). b) Even without surface BB, the work function can be reduced by molecular and bond dipoles (cf. Section 6). c) Upward surface band bending depends critically on the bulk doping concentration that also affects the surface dipole contribution (cf. Section 7).

near the i/o interface, which can even be laterally localized, whereas global effects are set by the material, such as the position of the Fermi level, or happen on large scales, such as charge transfer from the bulk to the surface.

We review our recent work on well-defined modifications of two of the lowindex $\mathrm{ZnO}$ surfaces that alter the surface potential and surface band bending with particular focus on this interplay of local (e.g., chemical bonds) and global properties of our samples (e.g., work function). To do so, we use a combination of state-of-the art density-functional theory and one- and two-photon photoelectron (1PPE and 2PPE) spectroscopy to characterize the changes to the surface and interface electronic structure. After briefly introducing fundamentals on energy level alignment, work function, and surface band bending (section 2.1) and current understanding of the surface electronic structure of $\mathrm{ZnO}$ (section 2.2), we present three exemplary model systems exhibiting up- and downward surface BB or flat band conditions (cf. Fig. 1). These are analysed with regard to the impact of interfacial charge transfer due to adsorption, molecular, and bond dipoles on the energy level alignment and work function at $\mathrm{ZnO}$ surfaces as outlined below.

We first take a detailed look at the effect of hydrogen adsorption on the electronic properties of $\mathrm{ZnO}(10 \overline{1} 0)$ with particular focus on the local changes to the surface potential and their impact on macroscopic properties, such as the sample work function [8]. We find that, in the low-coverage regime, 
hydrogen adsorption preferably leads to the formation of $\mathrm{O}-\mathrm{H}$ bonds and a concurrent, strong reduction of the work function and buildup of metallic surface state intensity (Fig. 1a). Both effects attenuate around 18\% O-H coverage as $\mathrm{Zn}-\mathrm{H}$ formation becomes energetically competitive. The maximum work function reduction of up to $0.6 \mathrm{eV}$ traces back to the electron transfer from hydrogen to $\mathrm{ZnO}$. It is connected to a strong downward surface $\mathrm{BB}$, which is confined to few $\AA$ as illustrated in Fig. 1a). As these potential minima are also laterally localised, the maximum surface BB contribution $\Delta \Phi_{\mathrm{BB}}$ around these minima is larger than the global work function change $\Delta \Phi_{\text {tot }}$, which is averaged over a large surface area. Moreover, we show that these local changes to the surface potential have a strong influence on the optical properties of $\mathrm{ZnO}$ surfaces: The local potential minima, caused by $\mathrm{O}-\mathrm{H}$ formation, host a very stable sub-surface bound exciton when the sample is photoexcited [9].

Then, we examine the influence of a small (dipolar) organic molecule, pyridine $\left(\mathrm{C}_{5} \mathrm{H}_{5} \mathrm{~N}\right)$, on the surface electronic structure. At full monolayer coverage, these molecules reduce the work function severely, by $2.9 \mathrm{eV}$ down to only $1.6 \mathrm{eV}$ [10]. Comparison of theory and experiment reveals that the molecules bind to every second zinc atom at the $\mathrm{ZnO}(10 \overline{10})$ surface and unveils the origin of the strong work function reduction: Both the permanent dipole moment of the pyridine molecules and the charge redistribution across the i/o interface equally contribute to this effect and lead to a massive work function reduction in the absence of surface BB (Fig. 1b)). Contrary to other molecules with a comparable potential to reduce work functions, the negative EA of pyridine prevents Fermi level pinning of unoccupied states of the molecule.

Furthermore, we discuss the adsorption of the organic acceptor tetrafluorotetracyanoquinodimethane (F4TCNQ) on the $\mathrm{ZnO}(000 \overline{1})$ surface $[11,12]$. Contrary to pyridine on $\mathrm{ZnO}(10 \overline{1} 0)$, the work function increases for F4TCNQ adsorption and reaches a change of $+1.4 \mathrm{eV}$ at full monolayer coverage. This work function increase is ascribed to long-range charge transfer from the $\mathrm{ZnO}$ bulk to the acceptor. Due to the buildup of space-charge regions and the associated long-range surface BB, the total charge transfer to F4TCNQ is small and remains below the detection limit of photoemission experiments.

In this Perspective, we put particular emphasis on the distinction between local and global aspects of the energy level alignment, which are crucial for energy transfer or charge separation processes. Our results illustrate how the fragile balance of charge distributions at i/o interfaces affect the work 
function. The broad spread of work function changes from $\Delta \Phi=-2.9 \mathrm{eV}$ to $+1.4 \mathrm{eV}$, and the multitude of responsible mechanisms (charge transfer, molecular dipole moment, surface BB etc.) for the three selected examples show that energy level alignment at ZnO-based interfaces is both difficult to predict and to explain. However, the number of competing mechanisms determining the energy level alignment also provides numerous ways of manipulating work functions and thereby controlling energy level alignment with great precision.

\section{Background}

\subsection{Energy level alignment and work function at inorganic/organic inter- faces}

To predict the level alignment for a certain system, the simplest approximation is to take the gas phase or bulk electron affinity (EA) and ionization energy (IE) of the molecule and adjust them to the vacuum level $\left(E_{\mathrm{vac}}\right)$ of the inorganic semiconductor and, thus, to its VB and CB (Fig. 2). This approach often fails to describe the interfacial energy levels, as it not only disregards difference in the surface electronic structure from the bulk, but also neglects possible changes due to bond formation and charge transfer between the organic and the inorganic components at the interface that can strongly affect the energy level alignment [13].

In reality, the situation is much more complex and has been extensively discussed in the literature $[14,15,16,17]$. Briefly, compared to the bulk of a solid, an unrelaxed surface exposes unsaturated bonds towards the vacuum. Although reconstructions can minimize the energy of the surface, a step in the potential $U(z)$ along the surface normal (cf. Fig. 2) is created by the electron density that is leaking out. This step is often described as the surface dipole and strongly influences the work function of a solid: the minimum energy needed to take an electron from the Fermi level $E_{F}$ into the vacuum $\Phi=E_{\mathrm{vac}}-E_{\mathrm{F}}$. In addition to the surface dipole contribution, the surface potential is also determined by the many body interaction of an electron in the vacuum in front of the surface, which is, for instance, very pronounced in the case of metal image potentials [18, 19, 20, 21, 22]. At a certain distance from the surface plane, the vacuum energy $E_{\mathrm{vac}}(z)$ remains essentially constant and defines the surface work function $\Phi$ [14]. This material surface property is most relevant for the energy level alignment of adsorbates (Fig. 2) as discussed below. It should be noted that, for larger 
distances on the order of the sample dimension, the electron in the vacuum is less and less influenced by the surface dipole field and, eventually, the vacuum level approaches its (field-free) value at infinity $E_{\mathrm{vac}}(\infty)[14,16]$.

The surface charge distribution also causes surface band bending (BB) in the case of semiconductors, which exhibit shorter screening lengths than metals. In other words, surface BB is caused by a charge carrier density different at the surface than in the bulk due to surface states or surface reconstructions. Surface BB is inversely proportional to the bulk doping concentration and shifts the electronic bands at the surface either up or down relative to the bulk bands. It is noteworthy that the bulk doping ( $n$ - vs. $p$ type) does not unambiguously determine the character of the surface BB (up vs. down). In the case of the $\mathrm{Si}(111)$ surface, indeed, $n$-type doping goes along with an upward $\mathrm{BB}$, leading to an electron injection barrier and $p$-type doping results in a downward $\mathrm{BB}$, creating a hole injection barrier. Probably due to the great importance of silicon in technology and the multitude of scientific studies on this material, this behaviour is often assumed to be a general rule. However, this is not the case. The type of BB depends on the net charge distribution between the bulk of a crystal and its surface. If the surface states have a donor character, i.e., they (partially) donate electrons to the bulk, the potential is pulled downward at the surface. The opposite is true if the surface state accepts electron density from the bulk, which leads to an upward surface BB [23]. ${ }^{1}$ In this Perspective, we will present both of these cases for $\mathrm{ZnO}$ surfaces.

When atoms or molecules are adsorbed at a surface, they disturb the electronic and structural balance of the pristine surface. The system reorganizes such that it minimizes its energy, which can lead to new reconstructions and charge distributions. Compared to the bulk of the inorganic semiconductor, the surface may become more positively or more negatively charged, hence modifying the surface BB and enhancing or reducing the work function, respectively (cf. Fig. 2). As the alignment of the energy levels of the adsorbed species is strongly affected by a change of the work function, this modification of the surface dipole needs to be understood and utilized. Recent attempts to consciously tailor the work function of a system such that the

\footnotetext{
${ }^{1}$ In the case of $n$-doped $\mathrm{Si}(111)$, the mid-gap surface states have an acceptor character, leading to the upward BB discussed above. For $p$-doped $\mathrm{Si}(111)$ the mid-gap states are donor in character, and the situation is reversed.
} 


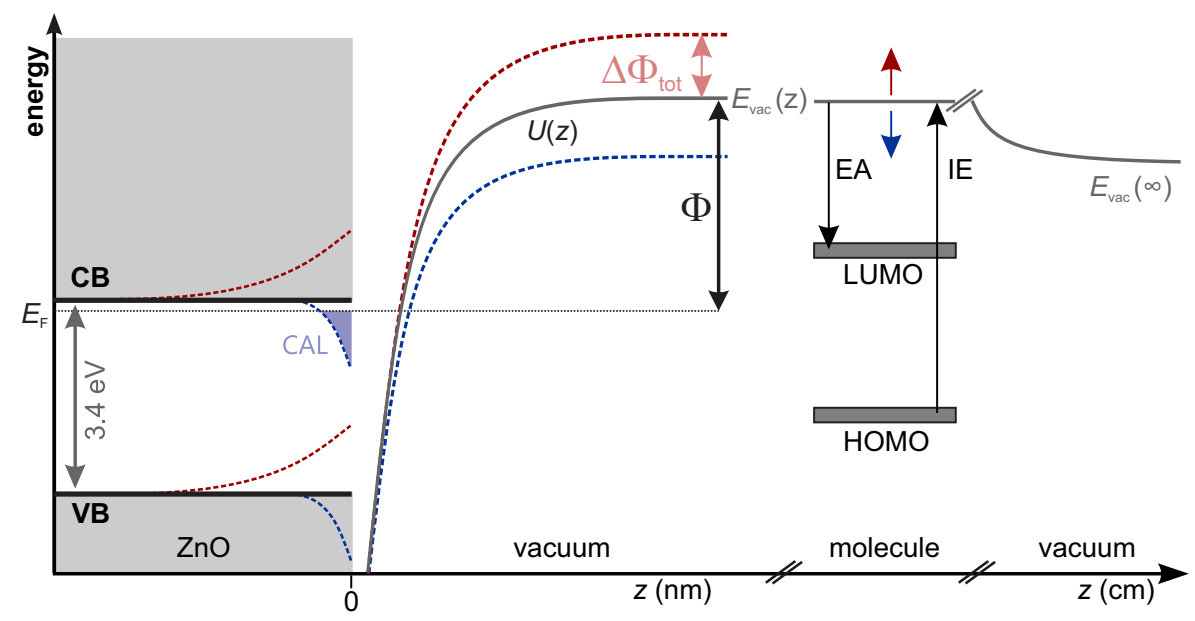

Figure 2: (color online) Simplified scheme of energy levels, potential $U(z)$, and work function $\Phi$ of the $\mathrm{ZnO}(10 \overline{10})$ surface and possible modifications by adsorption of electron donors (acceptors) in blue (red): Downward (upward) surface band bending is accompanied by a decrease (increase) of $\Phi$, respectively. Due to intrinsic $n$-type doping of the $\mathrm{ZnO}$ bulk, the downward surface band bending leads to the formation of a charge accumulation layer (CAL) at the surface.

level alignment is optimized [5] have used donor or acceptor molecules to deor increase the surface dipole. They, however, were limited by Fermi level pinning: The maximum achievable work function decrease, for instance, was determined by the EA, which defines the binding energy of the LUMO with respect to $E_{\mathrm{vac}}$. The LUMO is shifted down to $E_{\mathrm{F}}$ by the work function modification, and as soon as LUMO and $E_{\mathrm{F}}$ are resonant, charge transfer between the inorganic and organic semiconductor inhibits any further work function modification.

\subsection{Surface electronic structure of $\mathrm{ZnO}$}

The above-mentioned difficulties to predict and utilize energy level alignment at semiconductor surfaces have proven to be particularly tedious for metal oxides such as $\mathrm{ZnO}$ or $\mathrm{TiO}_{2}[24,25,26,27]$. While one of the most promising wide band gap semiconductors for optoelectronic applications [28], $\mathrm{ZnO}$ surfaces are notoriously difficult to prepare and characterize, both experimentally and theoretically $[29,30,31,32,33,34,35,36,37]$. ZnO exhibits room temperature luminescence, which is enabled by the comparably large bulk exciton binding energy $(60 \mathrm{meV})$. Effective electron and hole masses are only weakly affected by the electron-phonon interaction, although recently 
an intermediate hole polaron has also been observed in $\mathrm{ZnO}$ [38, 39]. Due to its great affinity for hydrogen $(\mathrm{H})$ interstitial and bulk oxygen defects $[40,41,42], \mathrm{ZnO}$ is "intrinsically" $n$-type doped.

$\mathrm{ZnO}$ also exhibits a plethora of nanostructures. Today, $\mathrm{ZnO}$ nanoparticles are already widely used for real applications, for example in chemical sensing [43]. Unfortunately, the microscopic origin of the activity of $\mathrm{ZnO}$ is still not fully understood. The reason for this lack of understanding is two-fold.

First, even the properties of pristine $\mathrm{ZnO}$ single crystal surfaces have severely challenged experimentalists for decades due to multiple surface reconstructions and defects that aggravate the reproducibility of surface condi-

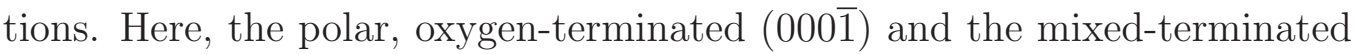
(1010) surface received particular attention, the latter being easier to control than the former [32]. Literature searches for the value of the work function of the low-index surfaces provide values scattered between 3.4 and $6.0 \mathrm{eV}$ $[44,45,46,47,11,48,49,50]$. Different preparation procedures and experimental conditions seem to have a major impact on the $\mathrm{ZnO}$ work function. Recently, the role of oxygen vacancies and hydrogen adsorption of the $\mathrm{ZnO}$ surface were increasingly discussed with regard to their role in energy level tuning. In particular, the adsorption of atomic hydrogen onto the $\mathrm{ZnO}(000 \overline{1})$ and $\mathrm{ZnO}(10 \overline{1} 0)$ surfaces can lead to the formation of a metallic surface state right below $E_{\mathrm{F}}$, which results from a downward bending of the $\mathrm{CB}$ minimum that is sufficiently strong to lead to a partial filling of the CB in the surface region $[51,52,53,9,8]$. Saturation of the $\mathrm{ZnO}(10 \overline{1} 0)$ surface with hydrogen, however, leads to a depletion of the $\mathrm{CB}$ and restores the semiconducting state $[54,8]$.

Second, quantitative, first-principles calculations for molecules adsorbed on surfaces are challenging for several reasons. DFT has become the firstprinciples method of choice for such adsorbate systems due to the computational efficiency of local and semi-local exchange-correlation functionals (augmented by appropriate van-der-Waals corrections $[55,56]$ ). However, the absence of surface polarisation (or image) effects $[57,58,59,60,61]$ and the presence of self-interaction errors $[62,63]$ limit the predictive power of these density functionals. This is particularly important for the transfer of charge $[12,64,65,66,67,68,69]$ and the associated change in the surface work function. Also, long-range charge transfer that leads to the buildup of space-charge regions could not, until recently, be included in DFT calculations due to the small size of the supercell $[70,12,71]$. To develop theoretical approaches further, experimental results are often used as benchmarks for 
theoretical methods, if theoretical benchmark data from high-level methods is not available. However, due to the aforementioned absence of reliable experimental benchmark data, the predictive power of a given calculation was low for ZnO-based adsorbate systems.

Fortunately, recent years have brought significant experimental and theoretical advancements. Progress in surface preparation and characterization techniques now enable experiments with well-defined and reproducible samples. Concomitantly, first-principles methodologies for polar oxides, their surfaces and adsorbates have advanced considerably. Combined, these developments provide an unprecedented potential for reaching a microscopic understanding of $\mathrm{ZnO}$-based organic-inorganic interfaces.

\section{Experimental details}

Pristine $\mathrm{ZnO}(10 \overline{1} 0)$ surfaces were prepared in situ under ultrahigh vacuum (UHV) conditions by repeated cycles of $\mathrm{Ar}^{+}$sputtering and annealing as described in detail in Ref. [8]. $\mathrm{H}_{2}$ was dosed using a background pressure of $6.7(2)$ mbar (corresponding to $0.5 \mathrm{~L} / \mathrm{s}$ ) with the sample in line of sight with a glowing tungsten filament leading to the adsorption of atomic hydrogen [8]. The data is, however, plotted as a function of $\mathrm{H}_{2}$ exposure, as quantification of the actual (atomic) hydrogen coverage is not possible. Comparison to theory allows for a rough coverage assignment (cf. chapter 5). Pyridine was adsorbed using a pinhole doser connected to a gas system that guided the pyridine vapour onto the cold sample surface. The coverage of the latter was determined using temperature programmed desorption as reported in Ref. [10].

For photoelectron spectroscopy (PES), we used an amplified femtosecond laser system working at a repetion rate of $200 \mathrm{kHz}$ and providing laser pulses (40 fs) with a photon energy of $1.55 \mathrm{eV}(800 \mathrm{~nm})$ that are used to drive an optical parametric amplifier to create pulses in the visible range. Near-UV light up to $6.2 \mathrm{eV}$ is subsequently generated by frequency doubling, tripling, and quadrupling. If the photon energy is large enough to photoionize the sample with one photon, occupied electronic states are probed in 1PPE (cf. Fig. 3b)). In contrast, if the photon energy is smaller than the work function of the sample, two photons are required to photoionize the sample and generate photoelectrons $(2 \mathrm{PPE})$. In the latter case, the photoelectron (PE) spectra also provide information about the normally unoccupied states of the 


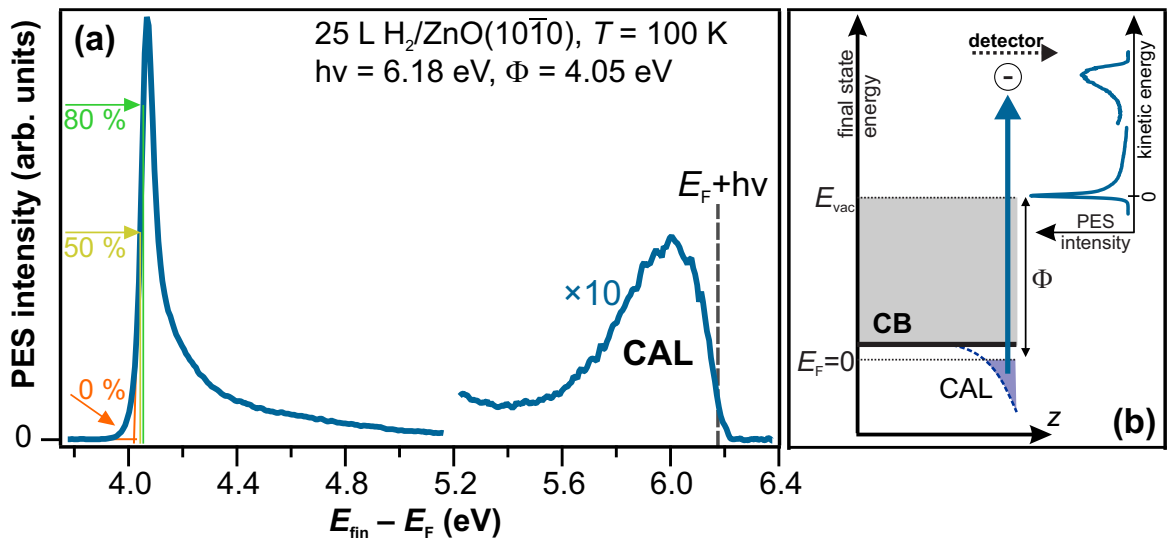

Figure 3: (color online) a) Exemplary 1PPE spectrum of $\mathrm{ZnO}(10 \overline{10})$ exposed to $25 \mathrm{~L}$ $\mathrm{H}_{2}$, plotted as a function of final state energy with respect to $E_{\mathrm{F}}=0$, i.e., photoemitted electrons from $E_{\mathrm{F}}$ have a final state energy equal to the absorbed photon energy (here: $6.18 \mathrm{eV}$ ). The high intensity at the low-energy cutoff results from secondary electrons with kinetic energies close to zero. This cutoff thus enables the direct measurement of the sample work function $\Phi$ as illustrated by panel b). Panel a) adapted with permission from Ref. [8]. Copyrighted by the American Physical Society.

sample, which are transiently populated by the first photon and emitted into the vacuum (i.e., excited above $E_{\text {vac }}$ ) by the second photon.

Time-resolved (TR) PES was performed in a pump-probe scheme: A first laser pulse (pump) photoexcites the sample as illustrated in Fig. 9. The subsequent equilibration dynamics are then monitored by a second laser pulse (probe), that photoemits the excited state population. Time resolution is achieved by changing the time delay between pump and probe pulse using a translational stage equipped with a retroreflector that varies the path length of the probe laser beam.

Photoelectrons are detected using a hemispherical photoelectron analyser with energy and momentum resolution (SPECS). The entrance cone of the analyser was extended such that the photoelectrons enter the field-free $\mu$ metal-shielded electron optics only $4 \mathrm{~mm}$ away from the sample surface, suppressing electric field effects from sample holder and sample edges. The Fermi energy was measured at the tantalum sample holder, which was in electrical contact with the sample. Charging and space-charge effects were ruled out by fluence-dependent measurements. 


\subsection{Determination of the work function}

Here, photoelectron spectra are either given as a function of the final state energy with respect to the Fermi energy

$$
E_{\text {fin }}-E_{\mathrm{F}}=\Phi+E_{\text {kin }}
$$

or as a function of the intermediate and initial state energy

$$
E-E_{\mathrm{F}}=E_{\text {kin }}-h \nu_{\text {probe }}+\Phi .
$$

As can be seen in the example in Fig. 3a), these photoelectron spectra expose a low-energy cutoff of the secondary electrons, which enables the direct determination of the sample (surface) work function: The barrier in the surface potential (cf. Fig. 2) blocks all photoexcited electrons with energies $E-E_{\mathrm{F}}<\Phi$ from leaving the sample, and only those with higher energies will be photoemitted with kinetic energies $E_{\text {kin }}$ (Fig. $\left.3 \mathrm{~b}\right)$ ). For samples with a homogeneous work function, this "filter" can be viewed as a step function in energy. The width of the low-energy cutoff in the experiment is then only determined by the energy resolution of the electron analyser (here $80 \mathrm{meV}$ ). Thus, the work function $\Phi$ can be read off the spectra at approximately 50$80 \%$ of the cutoff peak intensity, depending on the intensity distribution that needs to be convolved with the resolution function, as illustrated in Fig. 3a). Unfortunately, in the literature on (organic) semiconductor photoelectron spectroscopy, the work function determination often occurs at the low-energy onset of the secondary cutoff, i.e. at $0 \%$ (orange in Fig. 3a)). This leads, in the case of one well-defined, homogeneous work function of the sample, to a systematic underestimation by at least half of the experimental energy resolution.

For samples with inhomogeneous surfaces and a distribution of work functions, the situation is more complicated. Although the sample work function is, for $z \rightarrow \infty$, still a well-defined quantity, different local surface dipoles cause different barriers for electron emission. In how far these lateral variations of the potential influence the appearance of the low-energy cutoff strongly depends on the size and ratio of the corresponding surface areas as well as the distance and bias voltage at which the work function is measured $[72,73]$. In many cases the cutoff will be inhomogeneously broadened. Sometimes the photoemission spectra might even exhibit several edges [74]. The center of such a broad "cutoff" then provides an weighted mean work function in the probed area while its low-energy onset defines the minimum 
$\Phi$ observed (again, the experimental resolution should be considered). Regrettably, these distinctions are rarely found in literature, complicating the comparability of experimental values.

All experiments presented in this work showed narrow cutoffs on the order of the experimental resolution and thus provide absolute values of the homogeneous work function, which we extracted at the center of the lowenergy cutoff.

\section{Theoretical details}

\subsection{Density-functional theory calculations for surfaces and interfaces}

We use DFT to calculate the atomic and electronic structure of surfaces and molecular adsorbates. Surfaces are represented in the supercell or slab model, in which the surface unit cell is repeated periodically in directions perpendicular to the surface normal. In the direction of the surface normal, the surface is represented by a thin slab of material surrounded by vacuum. This supercell is then again repeated periodically in this spatial direction. The vacuum layer needs to be thick enough to prevent any interactions between the slab in the supercell and its periodic replica. If the surface dipole on one side of the slab differs from the other, a net dipole builds up across the slab model. In this case, the resulting dipole-dipole interactions between the slab and its periodic images have to be removed by dipole corrections [75].

The adsorbate-induced work function changes discussed in this Perspective are electrostatic in nature and are best illustrated by the Helmholtzequation

$$
\Delta \Phi_{\mathrm{tot}}=-\frac{e}{\epsilon_{0}} \frac{\mu}{A}
$$

where $e$ is the elementary charge, $\epsilon_{0}$ the vacuum dielectric constant, and $\mu / A$ the adsorption-induced change of the surface dipole density, with $A$ the surface area. In practice, we calculate the work function from the difference of the vacuum level to the Fermi level. Comparable to the analysis presented in [76], $\Delta \Phi_{\text {tot }}$ is then determined from the work function difference of two separate calculations, with and without adsorbate.

The adsorption-induced change of the dipole $\mu$ consists of different contributions, including the intrinsic molecular dipole moment, depolarization from the surrounding medium, bond and image dipole formation, charge transfer as well as potential BB. BB results from charge transfer due to the buildup of 
a space-charge region and can be long- or short-ranged. We denote the BB contribution to the work function change associated to long-range charge transfer $\Delta \Phi_{\mathrm{LR}}$. The remaining contributions are summed up in $\Delta \Phi_{\text {surf }}$ so that $\Delta \Phi_{\text {tot }}=\Delta \Phi_{\text {surf }}+\Delta \Phi_{\mathrm{LR}}$. We make the distinction between different ranges here, because long-range $\mathrm{BB}$ typically exceeds the size of tractable supercells in DFT. Our definition of short range is therefore everything that fits inside a supercell, i.e. a few nanometers. Long range then starts at a few nanometers and may extend up to micrometers. The inclusion of long-range BB in our supercell DFT calculations required method development that will be discussed below. The other contributions are captured in supercell DFT calculations that can be performed with standard first principles codes.

Until a few years ago, such DFT calculations for molecules on oxide surfaces were almost exclusively carried out with local or semi-local exchangecorrelation functionals due to their computational efficiency. It has since been recognized that the inherent self-interaction errors $[62,63]$ in these functionals limit their predictive power, particularly for the level alignment and the transfer of charge at interfaces [12, 64, 65, 66, 67, 68, 69]. Selfinteraction errors can be ameliorated in the computationally more expensive hybrid functionals that include a certain percentage of exact exchange. Such hybrid functionals have become computationally tractable for surface and surface-adsorbate systems due to advances in high-performance computing architectures and efficient exact-exchange implementations in common electronic structure codes. In recent years, the number of hybrid functional calculations for $\mathrm{ZnO}$ surfaces and $\mathrm{ZnO}$-based i/o hybrid systems has subsequently increased considerably [77, 78, 79, $12,80,10,37,81,82,8,83,84,85,86,87]$.

Ideally, one would want to go beyond the realm of DFT and use Green's function theories based on the screened Coulomb interaction to describe the quasiparticle band structure of these surface adsorbate systems. One such prominent method is Hedin's $G W$ approach [88]. $G W$ is particularly appealing for surface systems, because it correctly incorporates image effects $[57,58,59,60,61]$, which can be very pronounced for adsorbate states and critically influence the level alignment. While $G W$ has now become the method of choice for computing quasiparticle spectra in bulk materials [89, 90, 91, 92, 93, 94], conceptual [95] and numerical challenges remain for surfaces, interfaces and surface adsorbates. The adverse scaling of $G W$ with the number of atoms in the system and the slow convergence of quasiparticle energies with respect to the number of unoccupied states that are included in the calculation pose serve challenges to the 


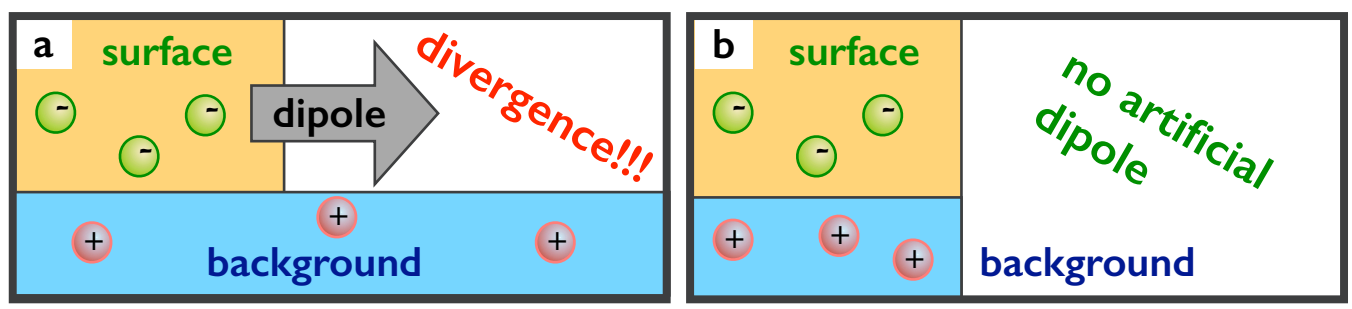

Figure 4: a) The introduction of negative charge into a periodic supercell requires a positive compensating background. The uniform background leads to a dipole that diverges upon increasing the amount of vacuum in the supercell. b) Confining the compensating charge to the extend of the slab in the surface supercell prevents the buildup of an undesired dipole.

tractable system size, which is easily exceeded by most surface adsorbate systems of interest. Nonetheless, an increasing number of $G W$ calculations for organic adsorbates on surfaces is being reported in the literature [96, 97, 98, 99, 100, 101, 102, 103, 104, 105, 106, 107, 108, 109].

\subsection{Charged surfaces and interfaces in the supercell method}

In certain cases, the surface unit cell might not remain neutral but will become charged. This can happen because defects form at the surface and donate or receive charge from the Fermi level. Or, the surface reconstructs in a way that does not obey the electron counting rule. Resulting excess electrons or holes will again be transferred to the Fermi level and enter the bulk or the near surface region. This charge rearrangement will build up space-charge regions that give rise to $\mathrm{BB}$ and alter the potential landscape at the surface. In this Perspective, we present two different scenarios: electron transfer to the $n$-type semiconductor $\mathrm{ZnO}$ that results in short-ranged downward $\mathrm{BB}$ at the $\mathrm{ZnO}$ surface, and electron transfer from $n$-type $\mathrm{ZnO}$ to the surface, which results in long-range upward BB whose magnitude strongly depends on the bulk doping concentration of $\mathrm{ZnO}$. In the following, we briefly summarize the methodology that we had to develop to make charged surfaces tractable in our DFT calculations and to add long-range electrostatic effects, such as space-charge regions and the associated BB to our supercell calculations. A more in depth discussion can be found in the original articles [70, 80, 12, 71].

Charged surfaces require a more elaborate treatment than neutral surfaces in DFT. Additional electrons or holes can easily be added to the supercell calculation. However due to the periodic repetition of the supercell, 
this is equivalent to adding an infinite amount of charge, which will lead to a divergence of the electrostatic energy. To prevent this divergence, a uniform background of opposite charge is usually added [110] to simulate the charged system by an effective neutral system. In a surface calculation, the additional charge is confined to the slab, whereas the homogeneous background also covers the vacuum region of the supercell (see Fig. 4a)). This creates an unphysical dipole across the supercell, which again leads to a diverging electrostatic energy for increasing supercell sizes. Post-processing correction schemes have recently been devised to extract surface energies at finite supercell size $[111,112]$. Here, we follow a different strategy. We confine the compensating background to have the same spatial extent as the extra holes or electrons. Instead of distributing the compensating charge homogeneously within the slab, we introduce point charges at atomic sites. The excess charge goes to the valence or conduction band, simulating free charge carriers, whereas the modified nuclear charge simulates the charged background. Overall the supercell remains neutral, and the problem of a diverging energy with increasing vacuum separation between the slabs is solved $[70,80,113]$.

\subsection{Coupling band bending to first-principles calculations}

The charged supercell approach permits us to study charge accumulation or charge depletion at surfaces due to defects, reconstructions or (molecular) adsorbates $[70,12,80]$. It does not, however, yet include a realistic description of the Fermi level and long-range charge transfer effects that give rise to the buildup of space-charge regions and the associated BB in semiconductors and insulators. An example of molecular adsorbate-induced BB is shown in Fig. 5a). In this illustration, the adsorbate is an acceptor with an acceptor state that resides below the Fermi level of an $n$-type semiconductor, such as $\mathrm{ZnO}$. Without anticipating the full story, we encounter this scenario for F4TCNQ on ZnO in Section 7.

During the adsorption process, charge is transferred from the semiconductor to the adsorbate. This charge transfer builds up a dipole that shifts the molecular state up in energy until it reaches the Fermi level. The dipole also changes the work function $\Phi$ of the surface by $\Delta \Phi$, as illustrated by the Helmholtz equation (eq. 3) in Section 4.1. Unlike metals, the charge transfer in semiconductors can be short- or long-ranged. Short-ranged charge transfer affects the near surface region and is either of local nature (hybridization, bond-formation, charge donation and back-donation) or of global nature (the 

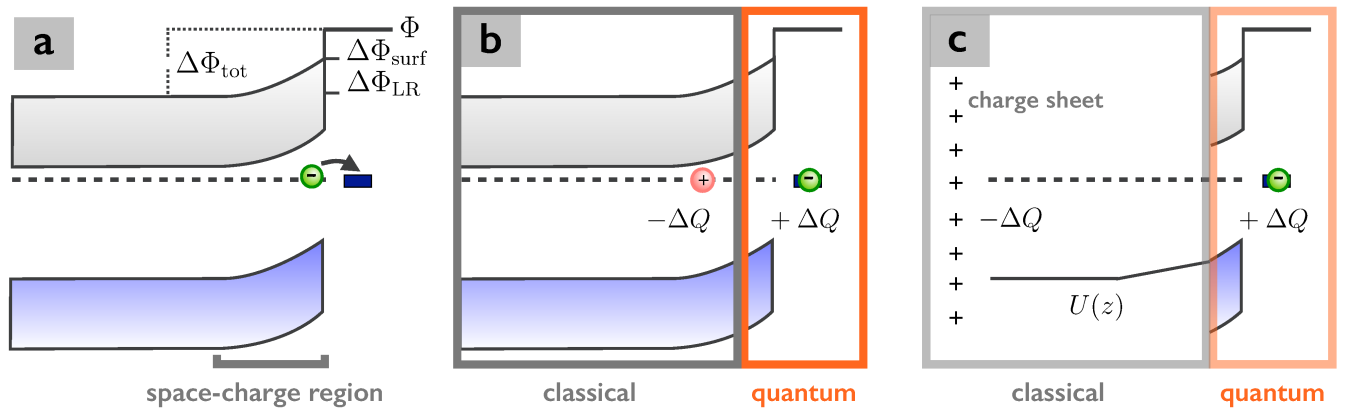

Figure 5: a) Schematic of adsorbate-induced surface BB at a semiconducting surface. The adsorbate is an acceptor, whose acceptor level is depicted by the dark blue bar. The charge $\Delta Q$ is transferred from the space-charge region of the semiconductor to the adsorbate giving rise to upward BB. b) In the CREST approach, the system is divided into two regions. The surface region is treated with quantum mechanical approaches and is coupled to a classical region. c) The classical region restores the correct electrostatic environment $U(z)$ using a charge sheet, which transfers charge to the quantum mechanical region.

Fermi level is above or below donor or acceptor states). In our F4TCNQ example in Fig. 5a), electrons are transferred from ZnO to F4TCNQ. Since $\mathrm{ZnO}$ is a semiconductor, this charge transfer builds up a space-charge region below the surface, which gives rise to $\mathrm{BB}$. For upward $\mathrm{BB}$ in $n$-type semiconductors, as is the case here, the extent of the space-charge region is inversely proportional to the bulk doping concentration and to the dielectric constant of the semiconductor.

For very high doping concentrations, the space-charge region will be small and is encompassed by the DFT supercell. In such cases, BB will be included in our DFT calculations and $\Delta \Phi_{\text {surf }}$ is equal to $\Delta \Phi_{\text {tot }}$. We show an example of such a situation in Section 5. However for low to moderate doping concentrations, the space-charge region is typically too large to be treated with DFT. For ZnO with a doping concentration of $10^{17} \mathrm{~cm}^{-3}$, for example, the space-charge layer would extend $100 \mathrm{~nm}$ into the bulk [12], while the tractable thickness of the slab in DFT calculations is at most a few nanometers. To include the effect of BB in our quantum mechanical calculations and to guarantee the correct amount of charge transfer, we developed a multiscale approach that divides the whole semi-infinite system into a classical region and a quantum mechanical region [71]. The classical region provides 
the correct electrostatic boundary conditions for the supercell and ensures that charge can flow from the bulk of the semiconductor to the surface, that is the supercell, as depicted in Fig. 5b). With this technique, we can quantify the long-range $\mathrm{BB}$ contribution $\left(\Delta \Phi_{\mathrm{LR}}\right)$ of the work function change and add it to $\Delta \Phi_{\text {surf }}$ to obtain $\Delta \Phi_{\text {tot }}$.

The Charge-Reservoir Electrostatic Sheet Technique (CREST) [71], shown in Fig. 5c), achieves the correct charge transfer by replacing the classical region with a charge sheet. The sheet is originally neutral, but it must contain mobile charges that can be transferred to the surface of interest. For convenience, we add this sheet to the supercell, sufficiently far away from the back side of the slab such that it does not interact directly with either side of the slab. As the charge $\Delta Q$ is transferred from the sheet to the surface, the remaining charge $-\Delta Q$ acts together with $\Delta Q$ at the surface as a plate capacitor and generates the electrostatic field that is normally provided by the space-charge region. Since for a given $\Delta Q$, and a given bulk doping concentration and dielectric constant of the semiconductor, analytic expressions for the amount of $\mathrm{BB}$ and its direction can be found in semiconductor text books [114], we can match the electrostatic potential generated by the sheet to the potential in the supercell. $\Delta Q$ and the position of the charge sheet are then determined self-consistently until $\Delta \Phi_{\mathrm{LR}}$ in the DFT calculation matches the prediction of the analytic formula. A certain portion of $\mathrm{BB}$ is included directly in the supercell. The remaining potential drop happens in the classical region. Contrary to the actual $\mathrm{BB}$ in a semiconductor, its shape $U(z)$ is linear, because we generate the potential using a plate capacitor. However, the precise form of $U(z)$ does not matter as long as it seamlessly matches the potential of the supercell and provides the correct potential drop.

The importance of surface BB in atomistic surface calculations is increasingly gaining recognition $[8,12,68,69,70]$. The CREST approach provides a robust and efficient way to include the effects of space-charge regions in common quantum mechanical calculations in the supercell approach.

\subsection{Computational details}

All calculations were performed using the Fritz Haber Institute ab initio molecular simulations (FHI-aims) code [115, 116, 117, 118], employing the Perdew-Burke-Ernzerhof (PBE) generalized gradient functional [119] and the Heyd-Scuseria-Ernzerhof hybrid functional (HSE) [120, 121]. We adjust the amount of exact exchange to 0.4 and denote this functional HSE* ${ }^{*}$ The longrange part of van der Waals forces, which are not accounted for in standard 
semilocal or hybrid functionals, were included by the vdW-TS scheme. [55] For $\mathrm{ZnO}$, the necessary parameters were calculated using the approach described for atoms in solids [122], yielding $C_{6}=46.0183, \alpha=13.7743, r_{0}=$ 2.818 for $\mathrm{Zn}$ and $C_{6}=4.45343, \alpha=4.28501, r_{0}=2.953$ for O. "Tight" defaults were used for grids and basis sets. The self-consistent field cycle was converged to $10^{-6} \mathrm{eV}$ for the total energy, $10^{-4} \mathrm{eV}$ for the electron density and $10^{-2} \mathrm{eV}$ for the sum of eigenvalues. The individual set-up of the supercell is discussed in corresponding sections. We optimized all geometries in $\mathrm{PBE}+\mathrm{vdW}$ by relaxing the atomic position until the remaining forces were smaller than $10^{-3} \mathrm{eV} / \AA$. To prevent dipole interactions between the slab and its periodic replicas, we used a dipole correction [75].

\section{Hydrogen adsorption on $\mathrm{ZnO}(10 \overline{1} 0)$ : Local and global effects to the surface potential}

Figure 3a) presents an exemplary photoelectron (PE) spectrum of $\mathrm{ZnO}(10 \overline{1} 0)$ that was exposed to $25 \mathrm{~L}$ of $\mathrm{H}_{2}$ in the presence of a glowing filament (cf. experimental section 3) leading to a submonolayer coverage of atomic hydrogen. It exhibits two features. First, secondary electrons close to the low-energy cutoff at $4.05 \mathrm{eV}$ final state energy allow us to extract the sample work function as described in Section 3.1; second, a peak at higher energies results from the emission of electrons from just below the Fermi energy $E_{\mathrm{F}}$, i.e, at energies within the band gap of bulk $\mathrm{ZnO}$. The second feature is absent for pristine $\mathrm{ZnO}$ and clearly shows that hydrogen adsorption leads to the buildup of (occupied) density of states close to $E_{\mathrm{F}}$ and thus a metallic character of the $\mathrm{H} / \mathrm{ZnO}(10 \overline{1} 0)$ interface. This charge accumulation layer (CAL) is analysed in more detail in Fig. 6, which shows the intensity increase of the CAL with increasing hydrogen exposure. At the same time, the work function of the sample decreases from $4.5 \mathrm{eV}$ down to $3.8 \mathrm{eV}$.

The clear reduction of the work function indicates that the $\mathrm{ZnO}(10 \overline{1} 0)$ surface is, after hydrogen adsorption, more positively charged than before, suggesting a donor character of adsorbed $\mathrm{H}$ similar to the aforementioned hydrogen interstitials in the bulk of $\mathrm{ZnO}$. If the observed work function reduction would only result from charge transfer from the adsorbed hydrogen to the $\mathrm{ZnO}$ crystal, the new charge distribution would not only lead to a change of $\Phi$, but also to a downward surface BB, as illustrated by the dashed blue curves in Fig. 2a) and Fig. 3b), in agreement with the observation of a CAL. Since the $\mathrm{ZnO}$ sample in our measurements is $n$-type doped, the 
electrons donated by $\mathrm{H}$ remain at the surface, leading to the buildup of a narrow space-charge region (or charge accumulation layer), leaving behind positively charged hydrogen atoms that cause the downward BB and work function reduction. However, for higher $\mathrm{H}$ dosages when the CAL intensity decreases, the situation becomes more complex, as discussed in more detail below.

To examine the microscopic origin of the work function change and CAL formation, we performed DFT calculations for several sub-monolayer coverages of $\mathrm{H} / \mathrm{ZnO}(10 \overline{1} 0)$. The $\mathrm{ZnO}(10 \overline{1} 0)$ surface was modelled using a $4 \times 4$ unit cell containing $16 \mathrm{ZnO}$ surface dimers. Since we expect the CAL/spacecharge region to be small, we attempted to include its entirety in our DFT supercell. We found that a slab thickness of 32 single-layers was necessary to capture the whole extent of downward BB in the most extreme case in which each surface oxygen is decorated by a hydrogen atom. Since for this system surface BB is entirely enclosed in our supercell (see Fig. 7), we did not employ the electrostatic schemes discussed in Section 4.3. All calculations were done assuming a constant $\mathrm{ZnO}$ doping concentration of approximately $10^{19} \mathrm{~cm}^{-3}$, which was modelled using the approach described in Section 4.2.

Next, we examined $\mathrm{H}$ adsorption more closely. Since it would be impossible to calculate all $2^{32}$ possible $\mathrm{H}$ configurations in our surface supercell with $16 \mathrm{ZnO}$ dimers, we simplify our approach. We instead focused on the question if, at some point during the dosage of hydrogen, the formation of $\mathrm{Zn}-\mathrm{H}$ bonds could energetically compete with the formation of $\mathrm{O}-\mathrm{H}$ bonds. We calculated the Gibbs energy for the addition of another hydrogen atom to a surface that is already pre-covered with a sub-monolayer of hydrogen exclusively adsorbed on oxygen. We considered O-H pre-coverages in a wide range from $0 \%$ to $94 \%$. The Gibbs energy of formation, $\Delta G$ was calculated separately for adsorption on $\mathrm{Zn}$ or $\mathrm{O}$ sites as

$$
\Delta G^{\mathrm{Zn} / \mathrm{O}}=E_{n+1}-E_{n}-E(\mathrm{H})-\mathrm{TS}
$$

where $E_{n+1}$ is the energy of the $4 \times 4$ supercell with $n$-many pre-adsorbed hydrogens and one additional hydrogen adsorbed on either $\mathrm{Zn}$ or $\mathrm{O}, E_{n}$ the energy of the supercell without the additional hydrogen, $E(H)$ the energy of a hydrogen atom, $T$ the temperature in the experiment $(100 \mathrm{~K})$ and $S$ the configurational entropy. We omit contributions from vibrational zero-point energies and estimate the configurational entropy by evaluating the number of possible configurations assuming that all configurations have the same 

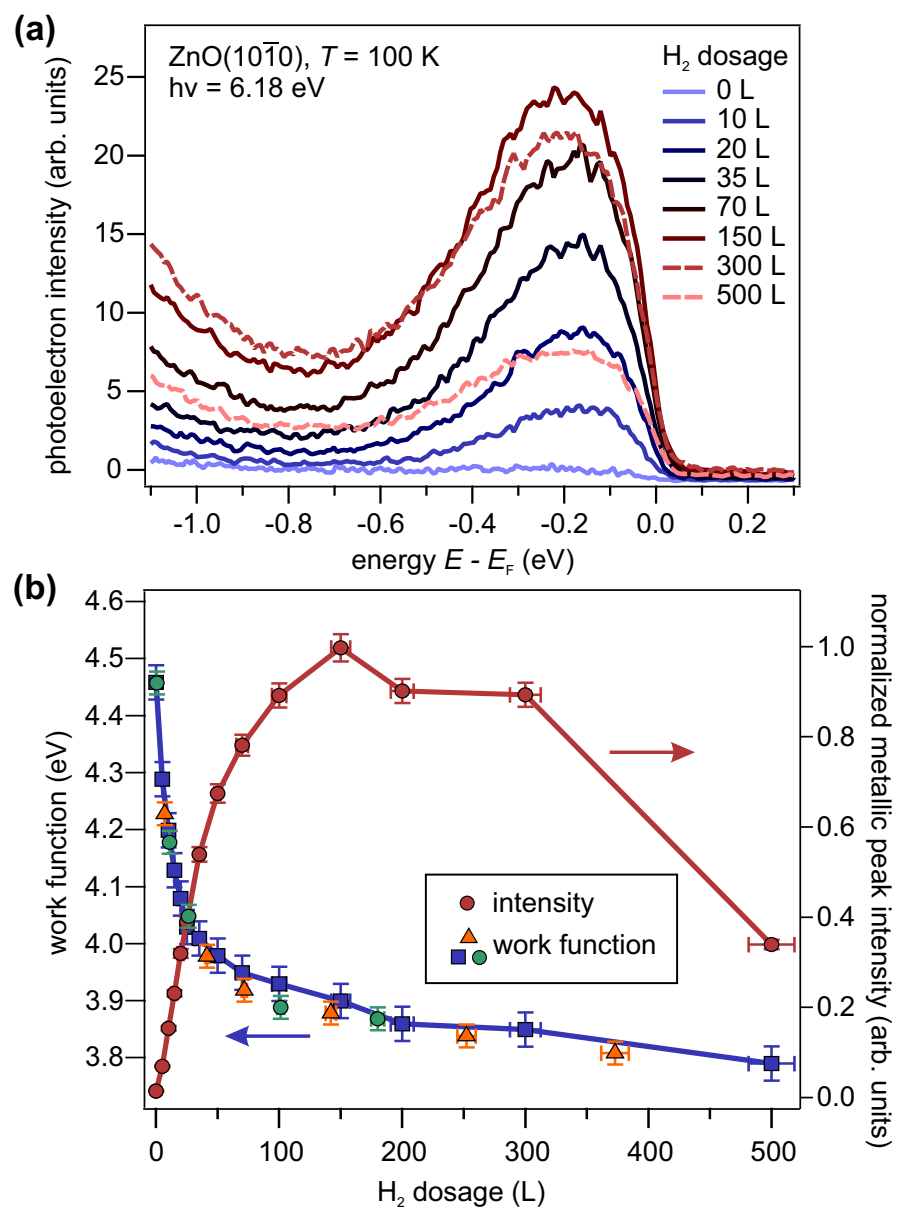

Figure 6: (color online) a) PE spectra of the CAL buildup as a function of $\mathrm{ZnO}(10 \overline{10})$ exposure to molecular hydrogen (cf. experimental section 3). b) CAL intensity change (right axis) and corresponding work function shift (left axis). Panel b) adapted with permission from Ref. [8]. Copyrighted by the American Physical Society. 

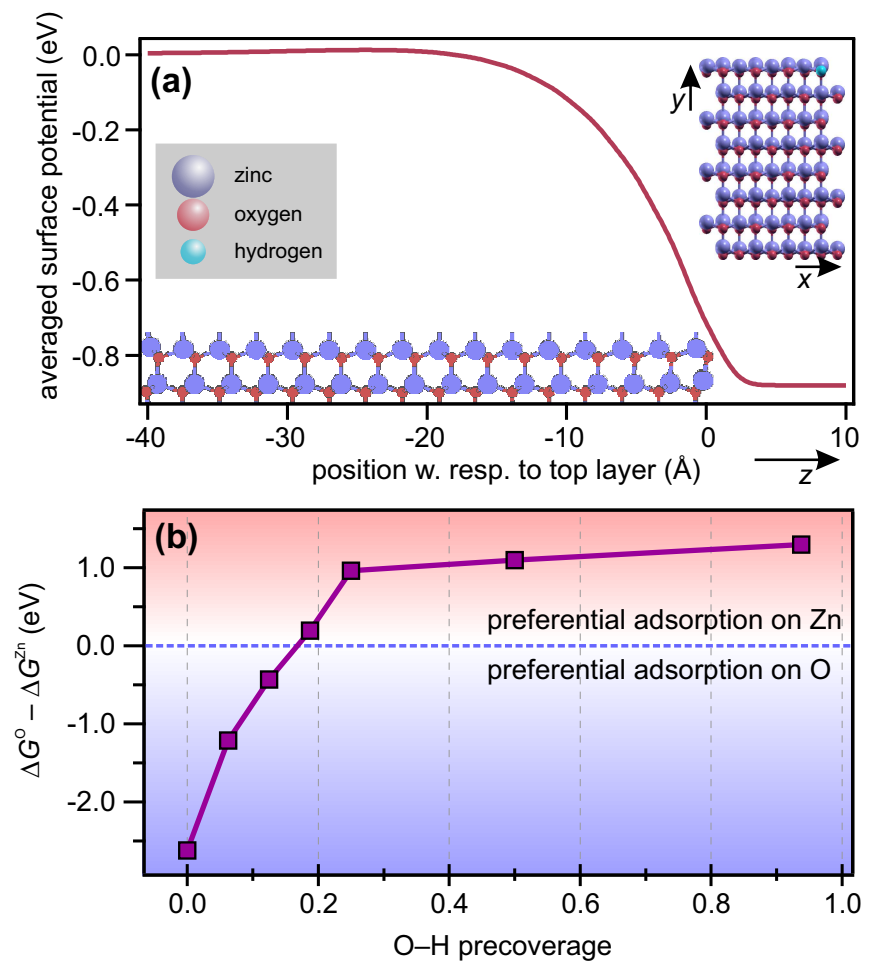

Figure 7: (color online) a) The adsorption-induced potential change along the surface normal reflects the surface BB due to a single hydrogen atom in a $4 \times 4$ unit cell (cf. inset) b) Energy balance of hydrogen adsorption on oxygen $\left(\Delta G^{O}\right)$ and on $\mathrm{Zn}\left(\Delta G^{\mathrm{Zn}}\right)$ as a function of O-H precoverage. For small numbers of surface hydroxyls, hydrogen adsorption on oxygen is more favourable and $\mathrm{Zn}-\mathrm{H}$ formation becomes competitive at a precoverage of $18 \%$. Figure adapted with permission from Ref. [8]. Copyrighted by the American Physical Society. 
energy [8]. We then considered pre-coverages of $n=1,2,3,4,6$, and $8 \mathrm{H}$ atoms in the unit cell with distributions that maximise the distance between $\mathrm{H}$ atoms. An additional hydrogen was introduced either on a $\mathrm{Zn}$ atom next to an $\mathrm{O}-\mathrm{H}$ bond or on an additional $\mathrm{O}$ atom.

Figure 7b) shows the Gibbs free energies for different $\mathrm{H}$ pre-coverages. We find that with increasing pre-coverage of $\mathrm{O}-\mathrm{H}$ bonds, the formation of further $\mathrm{O}-\mathrm{H}$ bonds becomes increasingly unfavourable, whereas the formation of $\mathrm{Zn}-\mathrm{H}$ bonds becomes more favourable. We attribute this behaviour to the amphoteric character of hydrogen, which acts as an electron donor on oxygen but as an electron acceptor on Zn. At a coverage of $18 \%$, hydrogen adsorption on zinc and oxygen becomes equally likely, and we expect mixed monolayers with $\mathrm{O}-\mathrm{H}$ and $\mathrm{Zn}-\mathrm{H}$ moieties to form around this coverage.

A complete picture of the $\mathrm{H}$-induced changes at the $\mathrm{ZnO}(10 \overline{1} 0)$ surface requires a microscopic description of the changes to the electrostatic potential $U$. Since $U$ enters the Schrödinger equation of our DFT calculations it affects the energies of the quantum mechanical states in the system and is indicative of BB. As a first step, we calculate the change of $U$ as

$$
\Delta U(x, y, z)=U^{\mathrm{ZnO}+\mathrm{H}}(x, y, z)-\left(U^{\mathrm{ZnO}}(x, y, z)+U^{\mathrm{H}}(x, y, z)\right)
$$

where $U^{\mathrm{ZnO}+\mathrm{H}}$ is the potential of the $\mathrm{ZnO}$ surface with adsorbed hydrogen, $U^{\mathrm{ZnO}}$ the surface without adsorbed hydrogen, and $U^{\mathrm{H}}$ the potential of a hypothetical, free-standing hydrogen layer in the same geometry as the adsorbed hydrogen atoms. The resulting $\Delta U$, averaged along $x$ and $y$, is shown in Fig. 7a) along the surface normal for a single hydrogen on an oxygen atom in a $4 \times 4$ supercell. The potential change exhibits downward band bending that is strongly localized along the surface normal and extends less than $20 \AA$ into the ZnO bulk. To our knowledge, this is the first DFT calculation of strongly confined downward surface BB in semiconductors and gives an important quantitative and microscopic confirmation of the prevalent view that charge accumulates in a thin surface layer in $n$-type materials (cf. Fig. 3a)) [51].

Figure 8 shows that the hydrogen-induced potential changes are also localised along the $x$ and $y$ directions. Figures 8b) and c) display the H-induced potential changes in the $(x-y)$-plane at $z=0 \AA$ and in the $(x-z)$-plane for a structure with $3 \mathrm{H}$ on $\mathrm{O}$ and $1 \mathrm{H}$ on a $\mathrm{Zn}$ site. $\Delta U$ varies strongly close to the $\mathrm{H}$ adsorption sites and is almost zero elsewhere. In this low coverage regime, changes to the surface electronic structure are strongly localized both laterally and along the $z$-axis. 

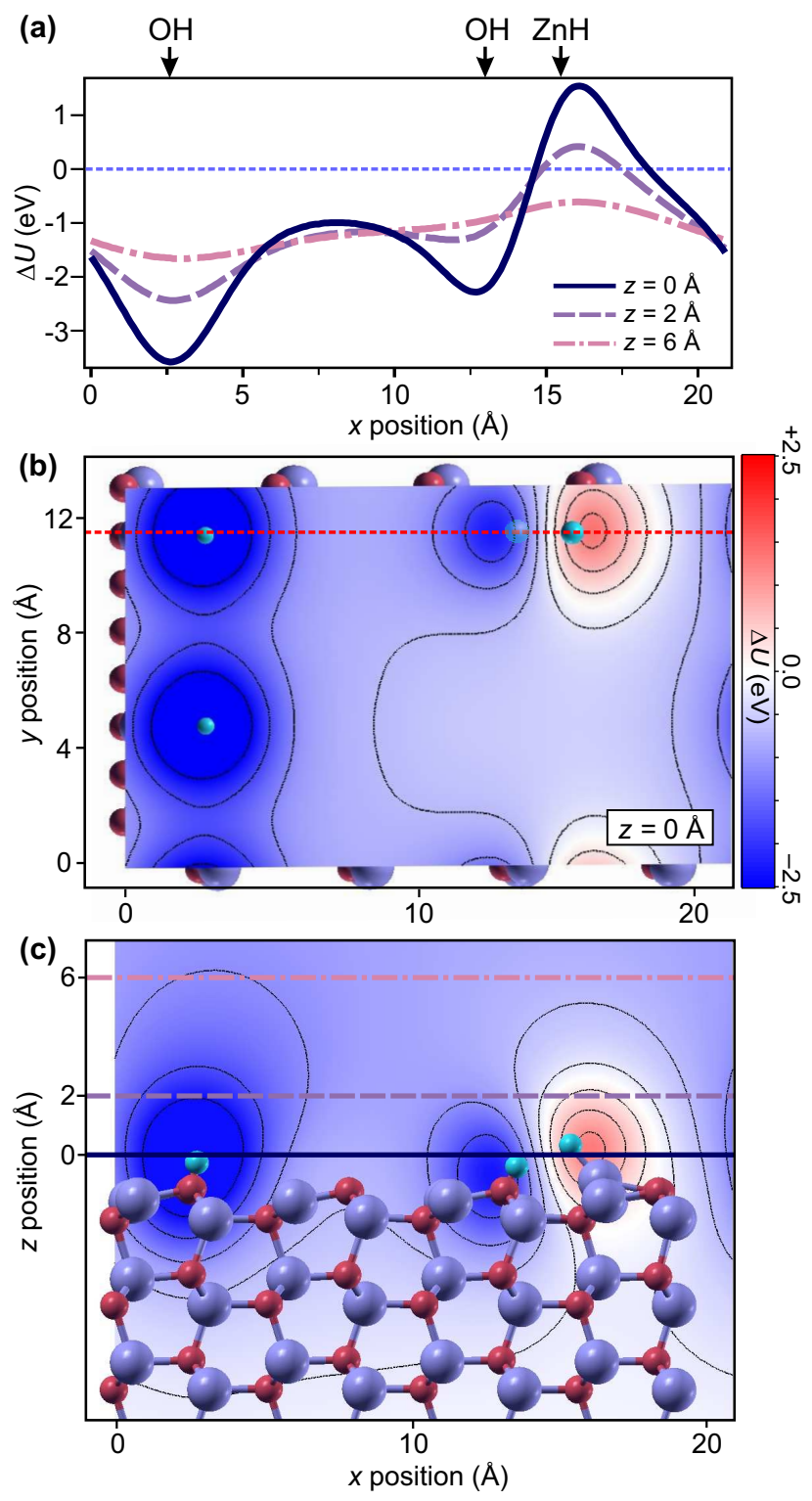

Figure 8: (color online) Hydrogen adsorption-induced potential change $\Delta U$, for a $\mathrm{H}$ adsorbed on $\mathrm{Zn}$ at a pre-coverage of $3 \mathrm{OH}$ groups. a) Dependence along the red dotted line indicated in b) for different distances from the surface. $z=0 \AA$ corresponds to the solid line shown in c). b) $\Delta U$ in false colors along the $(x-y)$-plane at $z=0 \AA$. c) $\Delta U$ shown for the $(x-z)$-plane cut along the line indicated in b). Isolines are drawn at $0.25 \mathrm{eV}$ increments of $\Delta U$. Adapted with permission from Ref. [8]. Copyrighted by the American Physical Society. 
Our microscopic calculations very illustratively show that hydrogen adsorption leads to very strong, very local perturbations of the potential energy landscape that reduce with increasing distance from the surface, eventually leading to the (global) work function change observed in the experiment. As discussed in detail in Ref. [8], the potential minima at the $\mathrm{O}-\mathrm{H}$ binding sites lead to a local, partial filling of the $\mathrm{CB}$ at the surface, resulting in the occupied surface state (or CAL) as schematically drawn in Fig. 3b). For low coverages, when the potential wells do not overlap, this should lead to a nearly linear increase of the CAL intensity. Once significant Zn-H formation sets in, the CAL intensity should decrease again. Both are indeed observed experimentally (cf Fig. 6b)), concurrent with a saturating reduction of the work function.

The observed corrugation of the surface potential in combination with the accumulation of electrons in the surface region can have severe consequences on the energy level alignment of adsorbed molecules in the vicinity of these sites: First, the potential minima will likely pull the energy levels of the adsorbed species downwards, which will have an impact on the electronic coupling and thus the charge separation probabilities. Second, the interfacial charge density may affect the screening of the Coulomb interaction at the interface and thereby influence charge transfer rates. However, such strong changes to the interfacial electronic structure may also give rise to entirely new excited states at the interface as discussed in the following.

Figure 9 displays the ultrafast dynamics launched by above band gap excitation of the $\mathrm{ZnO}(10 \overline{10})$ surface, investigated using femtosecond timeresolved PES. In these experiments, as discussed in detail in Section 3, the non-equilibrium dynamics caused by a first UV laser pulse $\mathrm{h} \nu_{\text {pump }}$ are monitored by a second, time-delayed laser pulse $\mathrm{h} \nu_{\text {probe }}$ that photoemits the transient electron population created by $\mathrm{h} \nu_{\text {pump }}$ (Fig. 9a)). Panel b) shows this photoinduced photoelectron intensity in false colors as a function of intermediate state energy (right axis) and pump-probe time delay (bottom axis). The measurement unveils (1) fast dynamics on femtosecond timescales at high energies that smoothly evolve towards (2) a long-lived contribution close to the Fermi energy. Detailed analysis of this data in Ref. [9] shows that the fast decay (1) can be attributed to the energetic relaxation of $\mathrm{CB}$ electrons by scattering with high energy (optical) phonons in agreement with theoretical predictions [123] and previous experiments [50]. The long-lived dynamics (2), on the other hand, reflect the formation dynamics of sub surface-bound excitons (SX) within $\tau_{\mathrm{X}}=200$ fs. Clearly distinct from excitons in the bulk 
of $\mathrm{ZnO}$, the electronic state provided by the presence of the photogenerated hole is below the Fermi energy in the case of SX and can thus only be localized in the vicinity of the potential minima caused by the hydrogen adsorption. This peculiar property makes them not only a highly stable excited state of the system (below the Fermi energy all resonant electron relaxation channels are blocked), but also arises from the hydrogen-induced modification of the surface electronic structure.

As discussed in detail in Refs. [9, 8], the overall SX intensity increases with increasing $\mathrm{O}-\mathrm{H}$ density, as the number of $\mathrm{O}-\mathrm{H}$ sites is increasing. Their sub-surface character is reflected by the observation that the UHV-prepared, partially hydrogen-terminated $\mathrm{ZnO}(10 \overline{1} 0)$ surface can be exposed to air without losing the SX signal [124]. However, at hydrogen exposures larger than $150 \mathrm{~L}$, the signal due to surface excitons reduces again, when the H-induced potential minima start to overlap. We interpret this vanishing of the SX species as a consequence of the delocalization of electrons in the CAL that leads to the suppression of exciton formation due to free carrier screening of the Coulomb attraction between the electron and the hole in the exciton, which will be the subject of a forthcoming publication.

In sum, despite the simplicity of the hydrogen atom, its adsorption on the mixed-terminated $\mathrm{ZnO}(10 \overline{1} 0)$ surface is highly complex with regard to its influence on the surface electronic structure and possible excitations therein. Depending on its adsorption site, hydrogen may lead to very severe, very local changes to the surface potential that become equal in the far field, where the work function is defined. The local change to the potential, which in itself can cause quite remarkable phenomena as the formation of a sub-surface excitonic species discussed above, the spatial dependence of $\Delta U(x, y, z)$ may also have strong influence on the energy level alignment of co-adsorbed molecules. As discussed in the introduction, in the most simple approximation, gas phase energy levels align with respect to the vacuum level of the system, which is defined by the sample work function $\Phi$. If the latter results from a surface potential that is homogeneous along the surface, the system can be (as is usual) regarded as a 1D problem in the $z$ direction with one surface dipole that shifts the energy levels down (or up). However, if the work function is a result of a spatially inhomogeneous potential with a different $z$ dependence at different sites on the surface, the situation is more complicated. The energy levels of the molecule will align according to the local potential, possibly leading to a different energy level alignment at different adsorption sites. This may cause an inhomogeneous broadening of the involved states or, if 


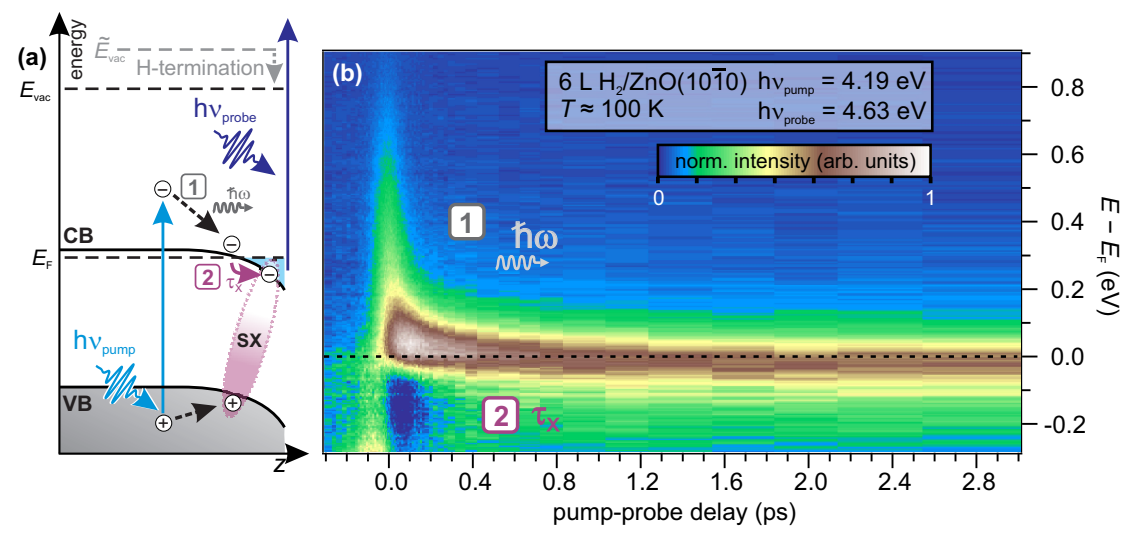

Figure 9: (color online) a) Experimental scheme for time-resolved PE spectroscopy and elementary processes: (1) Electron relaxation dynamics and (2) formation of sub-surfacebound excitons. b) Representative time-resolved PE measurement of $\mathrm{ZnO}(10 \overline{1} 0)$ surface. False colors display the photoinduced change of PE intensity (constant background subtracted). Excited electrons at high energies decay in femtosecond timescales, however the dynamics slow for lower energies. Remarkably, photoinduced PE intensity builds up below the Fermi energy $\left(E-E_{\mathrm{F}}=0\right.$, dashed line), indicating the creation of new states below $E_{\mathrm{F}}$ within $200 \mathrm{fs}$, which occurs due to surface exciton formation. Adapted with permission from Ref. [9]. Copyrighted by the American Physical Society.

distinct adsorption motifs are present, the occurrence of several peaks in the spectra. $^{2}$

The complexity of the $\mathrm{H} / \mathrm{ZnO}(10 \overline{1} 0)$ interface, in terms of local changes to the surface potential with strong effects on energy level alignment and dynamics, served as an example for the significant influence of seemingly weak perturbations of an inorganic semiconductor surface. In the next section, we discuss how adsorption of a polar molecule, pyridine, can alter the work function of $\mathrm{ZnO}$ even more strongly with particular focus on the limiting boundary conditions for work function modifications.

\section{Giant work function reduction at the Pyridine/ $\mathrm{ZnO}(10 \overline{10})$ in- terface}

As discussed in the introduction, the work function manipulation of surfaces by organic molecules has often been limited by the effect of Fermi level

\footnotetext{
${ }^{2}$ Naturally, such effects strongly depend on the size of the co-adsorbed species in relation to the distance between hydrogen adsorption sites and the size of the potential wells.
} 
pinning at the HOMO or LUMO of the molecules that were used for the work function modification. We circumvented this difficulty by using pyridine, an aromatic amine with a gas phase dipole moment of $2.2 \mathrm{D}$. Due to its negative EA (i.e., the LUMO lies above the vacuum level), no Fermi level pinning can occur for this molecule, enabling an enormous reduction of the sample work function as shown in the following.

Pyridine forms highly ordered structures on various polar and non-polar $\mathrm{ZnO}$ surfaces $[125,126]$. We use thermal desorption spectroscopy to determine the coverage of pyridine on the $\mathrm{ZnO}(10 \overline{1} 0)$ surface in units of monolayers [10]. As in the previous section, we monitor the coverage-dependent changes to the sample work function using the low-energy cutoff of the photoelectron spectra. Adsorption of 1 monolayer leads to the remarkable reduction of the $\mathrm{ZnO}$ work function by $\Delta \Phi=-2.9 \mathrm{eV}$ to a minimum value of $\Phi_{\min }=1.6 \mathrm{eV}$, which can be extracted from the blue spectrum in Fig. 10a). The evolution of the sample work function as the sample is heated is depicted in the main panel. Note that the observed work function change is only indirectly thermally induced; the elevated temperatures lead to the desorption of pyridine from the $\mathrm{ZnO}$ substrate and concurrently, the work function rises.

To understand the microscopic origin of this unusually large work function reduction, we performed DFT calculations for pyridine on the $\mathrm{ZnO}(10 \overline{1} 0)$ surface. The $\mathrm{ZnO}$ substrate was modelled by $8 \mathrm{ZnO}$ layers in the periodic slab. The atomic positions of the molecule and the top $4 \mathrm{ZnO}$ layers were relaxed, while the bottom 4 layers were fixed to their bulk positions. To model the $n$-type nature of the $\mathrm{ZnO}$ samples, we used an electron doping concentration of $4 \times 10^{16} \mathrm{~cm}^{-3}\left(10^{-6} e^{-} / \mathrm{O}\right.$ atom $)$ and the approach described in Section 4.2. We verified explicitly that higher doping concentrations up to $4 \times 10^{19} \mathrm{~cm}^{-3}$ give identical results within $10 \mathrm{meV}$ for both the adsorption energy and the interface dipole. Since pyridine does not induce charge transfer and $\mathrm{BB}$ on $\mathrm{ZnO}$, we did not use the CREST method.

For a single molecule at low coverage, we only find one stable geometry, shown in Fig. 10b). Pyridine adsorbs upright with the nitrogen atom located directly above a surface Zn atom and the aromatic plane oriented along the (1120)-direction, in agreement with a NEXAFS study of Walsh et al. for $0.1 \pm 0.05$ monolayer [127], except for a slightly larger tilt angle (theory $15^{\circ}$, experiment $10^{\circ}$ ). Our calculations show that increasing the coverage $(\Theta)$ does not affect the tilt angle. 


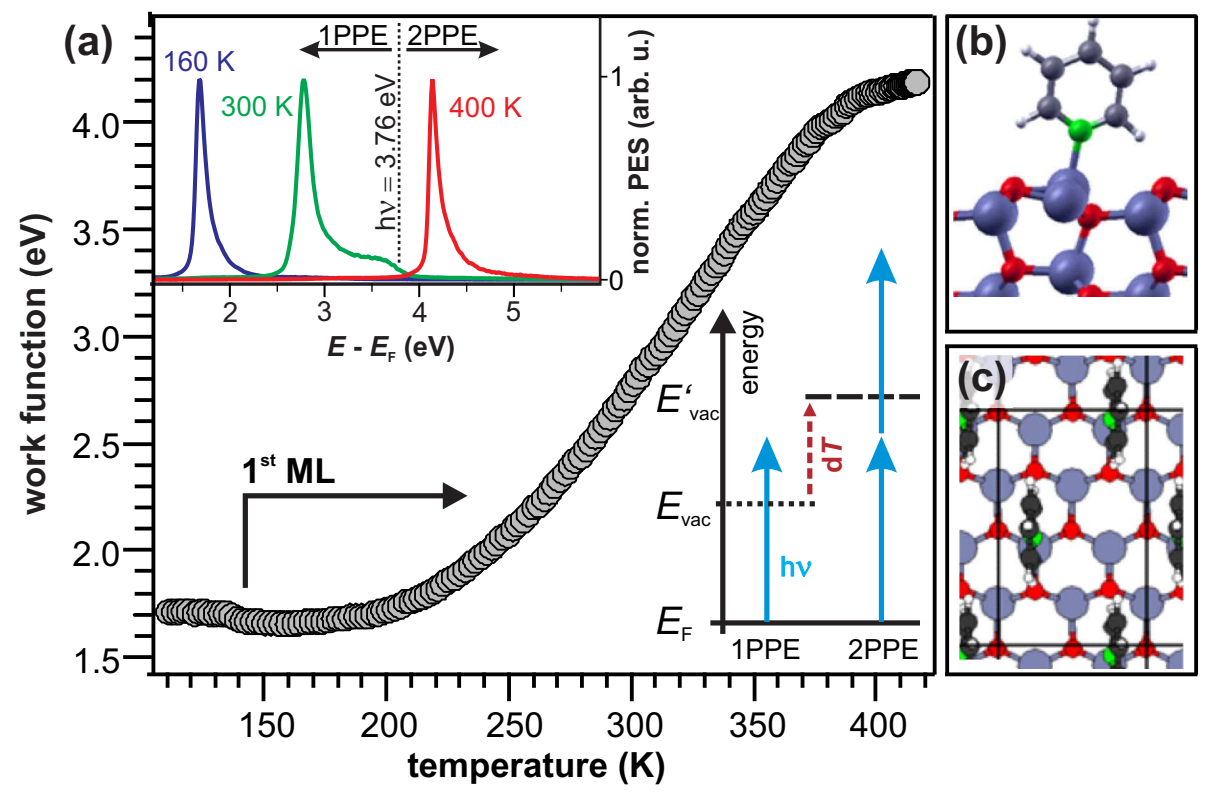

Figure 10: (color online) a) Work function evolution as a function of temperature during pyridine desorption from $\mathrm{ZnO}(10 \overline{1} 0)$ as measured by $1 \mathrm{PPE}$ and $2 \mathrm{PPE}$ using $\mathrm{h} \nu=3.76 \mathrm{eV}$ (bottom inset). The top inset depicts exemplary PE spectra. b) and c) show the calculated adsorption motif of pyridine calculated by DFT. Reproduced from [J. Chem. Phys. 139, 174701 (2013)], with the permission of AIP Publishing. 
The binding energy per unit area is

$$
E_{\mathrm{Ads}}=\left(E_{\mathrm{Sys}}-E_{\mathrm{Mol}}-E_{\mathrm{Slab}}\right)
$$

with $E_{\text {Sys }}$ being the energy of the combined pyridine/ZnO system, $E_{\text {Mol }}$ the energy of the free molecule in the gas phase and $E_{\text {Slab }}$ the isolated $\mathrm{ZnO}(10 \overline{10})$ surface. Figure 11c) shows the binding energy divided by the area per molecule. A pronounced minimum is found at a layer density corresponding to 1 pyridine / 2 surface Zn atoms, which we will henceforth adopt as full monolayer coverage, $\Theta=1.0$. The corresponding geometry is depicted in Fig. 10c). Further increasing the pyridine density will destabilize the layer, and the formation of a second layer, which is not in direct contact with the substrate (whose energy is shown in Fig. 11c) as an open star), will be favoured. By exploring different packing motifs for the second monolayer, we find several different minima with different dipole orientations within an energy range of $40 \mathrm{meV}$. Based on this theoretical information and the experimentally observed saturation of the work function near $\Theta=1.0$, we speculate that the second layer grows amorphously and does not exhibit a net dipole moment.

To compare the experimentally and theoretically determined work function changes, we plot the measured change of $\Phi$ from Fig. 10a) as a function of coverage in Fig. 11d) (open circles) with the DFT-PBE+vdW values (solid squares). We have ascertained that the HSE* DFT functional changes $\Delta \Phi$ by less than $5 \%$. Clearly, both data sets coincide for pyridine coverages below 1 monolayer. The theoretical data point at $\Theta=2$ monolayer illustrates a scenario, in which more pyridine molecules were forced into the monolayer, leading to a molecular density twice as high, while the dashed line shows the expected behavior for amorphous (i.e., disordered with regard to molecular dipoles) multilayer growth. Comparison to the experimental data that ranges up to 1.1 monolayers strongly supports the amorphous layer hypothesis.

Fig. 11d) also depicts two different contributions to the work function reduction, the independently calculated dipole of a hypothetical, free-standing pyridine layer $\Delta \Phi_{\text {Mol }}$ (red circles) and the remaining dipole resulting from the adsorption of this layer $\Delta \Phi_{\mathrm{Ads}}=\Delta \Phi-\Delta \Phi_{\text {Mol }}$ (blue triangles). $\Delta \Phi_{\text {Ads }}$ includes all adsorption-induced contributions of the pyridine layer on the $\mathrm{ZnO}$ substrate, including possible electronic responses in the inorganic semiconductor due to formation of image dipoles, for example. Up to 0.5 monolayer, they are equal, nearly linearly decreasing as the coverage increases. 
For larger pyridine coverages, however, the change of $\Delta \Phi_{\text {Ads }}$ becomes less pronounced while the molecular dipole contribution continues to contribute nearly linearly. As discussed in detail in Ref. [10], this results from charge redistribution due to covalent bond formation between $\mathrm{ZnO}$ and pyridine.

The observed substantial reduction of the $\mathrm{ZnO}$ work function by $\Delta \Phi=$ $-2.9 \mathrm{eV}$ is currently, to our knowledge, only exceeded by adsorption of alkali atoms on various surfaces $[128,129,130]$ and could not, so far, be reached by other organic molecules. This uniqueness of the pyridine molecule results from the concurrence of three advantageous properties that cooperate in reducing the work function [10]:

1. the permanent dipole moment of the molecule,

2. its tendency to form strong, polar covalent Zn-N bonds,

3. its negative EA.

Many organic molecules exhibit properties 1 and 2, for example dyes that use pyridine docking groups [131]. However, in most cases, these molecules have a positive EA, that is, their LUMO lies below the vacuum level as depicted in Fig. 11a). This attribute leads to a maximum reduction of the work function to $\Phi_{\min }$, which directly corresponds to the EA of the molecule. In this case, the LUMO has shifted to the Fermi energy, and any further work function reduction is exactly compensated by charge transfer at the i/o interface. This process of Fermi level pinning cannot occur if the molecule has a negative EA, that is, the LUMO lies above the vacuum level (cf. Fig. 11b)). Here, the work function could, given sufficiently strong interface dipoles, theoretically be reduced to zero. We conclude that, in the case of pyridine adsorption on $\mathrm{ZnO}(10 \overline{1} 0)$, the negative EA enables the observed, giant reduction by $2.9 \mathrm{eV}$ to $\Phi=1.6 \mathrm{eV}$, which is caused in equal parts by the permanent dipole moment and the polar covalent bond to the Zn atoms.

The adsorption-induced changes to the $\mathrm{ZnO}$ surface electronic structure are very different for atomic hydrogen and pyridine deposition. First, the work function reduction for the amine is twice as strong as for hydrogen. The dipole moment of pyridine and the covalent $\mathrm{Zn}-\mathrm{N}$ bond cooperatively contribute to $\Delta \Phi=-2.9 \mathrm{eV}$, which is made possible by the negative EA. Conversely, during hydrogen adsorption, electron transfer to $\mathrm{ZnO}$ gives rise to the work function change and not a permanent dipole. Second, the reduction of the work function for $\mathrm{H} / \mathrm{ZnO}(10 \overline{1} 0)$ is accompanied by a significant downward surface BB, whose extent is a few $\AA$ both lateral and normal to the surface. This is not the case for the pyridine/ZnO interface, for which we did not 


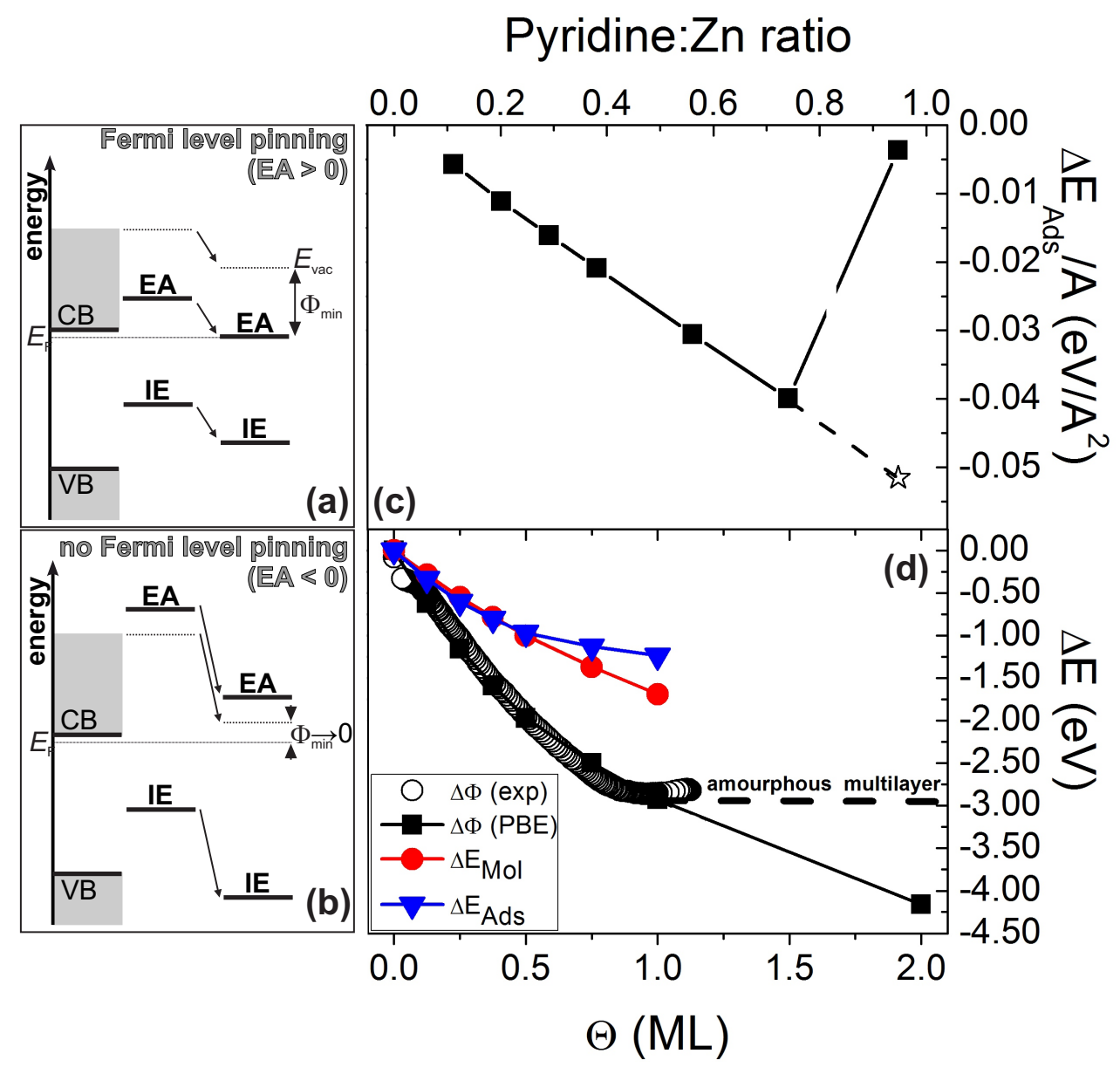

Figure 11: (color online) a) The work function reduction of molecules with positive EA is limited by Fermi level pinning. b) A negative EA generally allows for unlimited work function reduction, as Fermi level pinning cannot occur. c) Adsorption energy for pyridine on $\mathrm{ZnO}(10 \overline{1} 0)$, calculated by DFT. d) Comparison of the experimentally (open circles) and theoretically (solid squares) determined work function shift. Red and blue symbols illustrate the molecular and bonding contribution to the surface dipole, respectively. Panels c) and d) reproduced from [J. Chem. Phys. 139, 174701 (2013)], with the permission of AIP Publishing. 
observe BB of a similar extent. These two examples clearly demonstrate that different causes can lead to very significant work function reductions: charge transfer combined with short-range surface BB and permanent molecular dipole moments as well as polar bonds in the absence of surface BB. The next section is devoted to another, complementary example for the manipulation of the surface electronic structure: adsorption of the strong electron acceptor F4TCNQ (2,3,5,6-tetrafluoro-7,7,8,8-tetracyanoquinodimethane) on the polar oxygen-terminated $\mathrm{ZnO}(000 \overline{1})$ surface. It leads to an upward surface $\mathrm{BB}$, which extends significantly further into the bulk than the one discussed for $\mathrm{H}$ on $\mathrm{ZnO}$ in Section 5, as the Thomas-Fermi screening length is much larger in the absence of a charge accumulation layer.

\section{Charge transfer-induced upward surface band bending at the F4TCNQ/ZnO(000̄) interface}

In this last example, we present a situation for which molecular adsorption leads to upward surface BB and a pronounced work function increase. For $n$-type doping as typically found in $\mathrm{ZnO}$, upward $\mathrm{BB}$ is induced by acceptor states at the surface. These are filled by charge carriers from the Fermi level. Since these charge carriers can come from deep in the bulk of the semiconductor, the extent of the resulting space-charge region can be very large and depends strongly on the bulk doping concentration as illustrated in Fig. 12. This example is therefore distinctly different from the creation of downward BB for $n$-type conditions illustrated in Section 5.

To investigate the bulk-doping dependence, we performed DFT calculations for the electron acceptor F4TCNQ on the $\mathrm{ZnO}(000 \overline{1})-\mathrm{O}$ surface [12]. We adopted the $\mathrm{ZnO}(000 \overline{1})(2 \times 1)-\mathrm{H}$ surface structure for our calculations, in which every second row of surface $\mathrm{O}$ atoms is decorated with $\mathrm{H}$ atoms. This is thermodynamically the most stable structure in typical experimental growth conditions, according to our and previous studies [132, 36, 80]. We also previously showed that $\mathrm{ZnO}(000 \overline{1})$ surfaces with lower hydrogen concentrations (less than 50\%) may be stabilized by $n$-type bulk dopants in hydrogen-deficient environments [80]. We will not, however, consider these $\mathrm{H}$-deficient structures here. The $\mathrm{ZnO}$ surface was modeled by a slab with

four double layers and a $50 \AA$ vacuum separation. The top two layers were relaxed and the bottom end saturated by $\mathrm{H}$ atoms of charge $1.5 e$ at a relaxed geometry. 


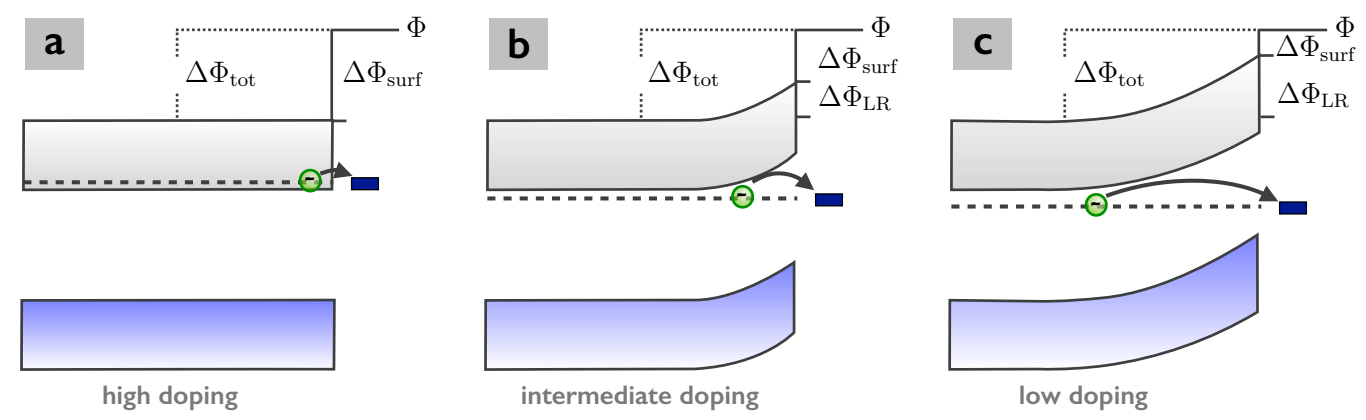

Figure 12: Schematic of the charge transfer-induced work function change at the F4TCNQ/ZnO interface for a) high, b) intermediate and c) low $\mathrm{ZnO}$ doping concentrations. $\Delta \Phi_{\text {surf }}$ includes adsorption-induced dipole contributions, which for F4TCNQ are relatively small [12], as illustrated by the small value in the low doping regime in panel c). The main contribution of $\Delta \Phi_{\text {surf }}$ at high-doping concentrations (panel a)) is an interface dipole that results from short-range charge transfer directly at the surface. This interface dipole is most easily defined in the flat-band limit of high-doping concentrations, in which $\mathrm{BB}$ is zero, and provides the necessary dipole to lift the acceptor state to the Fermi level. As BB increases due to the buildup of a space-charge region for intermediate to low doping concentrations, less charge is transferred, and the interface dipole contribution and concomitantly also $\Delta \Phi_{\text {surf }}$ reduces.

On the $\mathrm{ZnO}(000 \overline{1})(2 \times 1)$-H surface, F4TCNQ lies flat on the substrate with the cyano groups $(\mathrm{CN})$ located above the surface $\mathrm{H}$ atoms (see inset of Fig. 13). The four $\mathrm{CN}$ groups of $\mathrm{F} 4 \mathrm{TCNQ}$ are attracted by the surface $\mathrm{H}$ atoms and repelled by the surface $\mathrm{O}$ atoms. As a result, the molecule distorts slightly upon adsorption, placing the $\mathrm{N}$ atoms $0.4 \AA$ below the $\mathrm{F}$ atoms [12].

Our DFT-HSE* calculations predict an F4TCNQ-induced work function increase to $5.7 \mathrm{eV}$ for $n$-doped $\mathrm{ZnO}$ at full F4TCNQ coverage, which agrees well with the value of $5.9 \mathrm{eV}$ found in photoemission experiments [11]. With the methodology introduced in Section 4.3, we can now analyse these results in terms of BB. The total work function $\Phi$ and F4TCNQ-induced work function change $\Delta \Phi$ is fixed by the position of the F4TCNQ acceptor state before adsorption (or more precisely, before charge transfer) and does not depend on the doping concentration $N_{\mathrm{D}}$ of $\mathrm{ZnO}$, as shown in Fig. 12. However, the division of $\Delta \Phi$ into long-range $\mathrm{BB}\left(\Delta \Phi_{\mathrm{LR}}\right)$ and a surface dipole $\left(\Delta \Phi_{\text {surf }}\right)$ contribution depends strongly on $N_{D}$, as we demonstrate in the following.

In the Schottky-depletion-layer approximation $[133,114]$ the long-range 
$\mathrm{BB}$ contribution ${ }^{3}$ can be derived analytically

$$
\Delta \Phi_{\mathrm{LR}}=\frac{e^{2} \Delta Q^{2}}{2 \epsilon_{0} \epsilon N_{D}}
$$

where as before $e$ is the elementary charge, $\epsilon_{0}$ the vacuum permittivity, $\epsilon$ the static dielectric constant of the inorganic semiconductor, and $\Delta Q$ the charge per area transferred to the F4TCNQ acceptors. The corresponding change in total energy per area is given by [12]

$$
\Delta E_{\mathrm{BB}}=\frac{e^{3} \Delta Q^{3}}{6 \epsilon_{0} \epsilon N_{D}} .
$$

Since $\Delta \Phi$ is fixed by the position of the acceptor state before charge transfer, $\Delta \Phi_{\text {surf }}$ also acquires a doping dependence. This dependence is most easily illustrated by starting in the high doping regime shown in panel a) of Fig. 12. In this metallic regime, the bulk bands are flat, and $\Delta \Phi_{\mathrm{BB}}$ is zero. The usual adsorption-related effects discussed in previous sections create an adsorptioninduced dipole, but in the case of F4TCNQ, this is not enough to lift the acceptor state above the Fermi energy. Instead, the acceptor state would remain below $E_{F}$ in the band gap. Charge will now flow from the Fermi level to the acceptor state to create a charge transfer-induced dipole that will lift the acceptor state to the Fermi level. For simplicity, we include this dipole in $\Delta \Phi_{\text {surf }}$. Once the doping concentration reduces, the charge transferred to F4TCNQ is no longer screened perfectly by the electrons at the Fermi level, and BB will set in. This shifts the balance between global and local charge transfer as illustrated in Fig. 12 and reduces the charge in the acceptor state, as we also demonstrate numerically. We reiterate that our distinction between local and global is somewhat arbitrary, because it is impossible to unambiguously define the extent of the local region. The distinction is borne out of the necessity to include the multi-scale $\Delta_{\mathrm{LR}}$ corrections for doping regimes in which our supercell is clearly too small to incorporate the entirety of $\mathrm{BB}$.

\footnotetext{
${ }^{3}$ Here our definition of long range as anything that lies beyond the extend of the supercell becomes problematic for high doping concentrations. Since the extent of the space-charge region is inversely proportional to $N_{D}$, all BB will eventually be encompassed by the supercell for high enough $N_{D}$. At that point, $\Delta \Phi_{\mathrm{LR}}$ will become short-ranged, according to our definition. However, this is mostly a philosophical point, because a) CREST correctly takes care of the ranges, and b) $\Delta \Phi_{\mathrm{LR}}$ will be almost zero in this doping regime, as Fig. 12a) illustrates.
} 


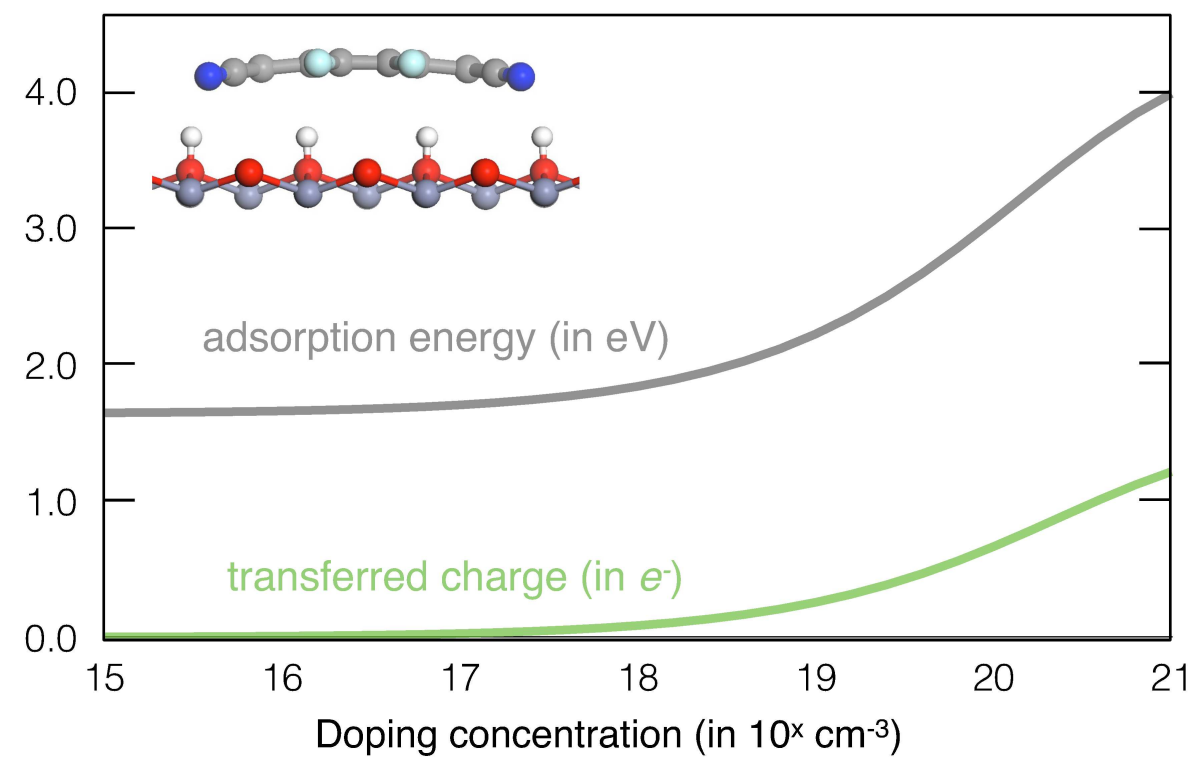

Figure 13: (color online) Adsorption energy (calculated according to eq. 6 and transferred charge $(\Delta Q)$ to F4TCNQ as a function of ( $n$-type) dopant concentration $N_{\mathrm{D}}$ for F4TCNQ/ZnO $(000 \overline{1})(2 \times 1)-H$. The atomic structure computed with PBE+vdW is shown in the inset [12].

Equations 7 and 8 clearly illustrate that the effect of $\mathrm{BB}$ is inversely proportional to the bulk doping concentration $N_{D}$. With our multi-scale approach presented in Section 4.3, we can now simulate F4TCNQ adsorption on $\mathrm{ZnO}$ samples with different bulk doping concentrations.

The results for the adsorption energy and the amount of transferred charge are shown in Fig. 13. Both indeed vary with $N_{D}$. For low $N_{D}$, the dependence is weak. Charge transfer is almost zero, and the adsorption energy assumes the value of $1.6 \mathrm{eV}$, a value we also find for a DFT calculation without any doping. In this low $N_{D}$ regime, $\mathrm{BB}$ is large due to the inverse dependence on $N_{D}$. The corresponding space-charge region extends far into the bulk, much farther than the H-induced downward BB discussed in Section 5. For $N_{D}=10^{17} \mathrm{~cm}^{-3}$, for example, it reaches $\sim 100 \mathrm{~nm}$ into $\mathrm{ZnO}$ [12]. Since the charge transfer $\Delta Q$ to F4TCNQ is small, the corresponding surface dipole contribution to the work function change $\left(\Delta \Phi_{\text {surf }}\right)$ is small. The whole work function change in this regime is therefore leveraged by $\Delta \Phi_{\mathrm{LR}}$. The $\mathrm{BB}$ contribution can be large despite the small $\Delta Q$ due to the inverse dependence on $N_{D}$. What matters is the ratio between $\Delta Q^{2}$ and $N_{D}$. 
The photoemission experiments of Ref. [11] reported a ZnO doping concentration of $10^{17}-10^{18} \mathrm{~cm}^{-3}$. According to Fig. 13 charge transfer is still less than 0.1 electron per F4TCNQ molecule in this range. However, the work function change remains large, and the calculated work function after adsorption is still $5.7 \mathrm{eV}$, as mentioned above. In the photoemission measurements of Ref. [11], the work function after adsorption is $5.9 \mathrm{eV}$, and the corresponding work function increase amounts to $1.4 \mathrm{eV}$. The photoemission measurements do not show a filled state near the Fermi energy that could be attributed to a filled LUMO state. Both theory and experiment therefore conclusively demonstrate that large work function changes with negligible charge transfer are possible due to the buildup of a space-charge layer and the associated BB $[12,11]$.

For higher doping concentrations, the extent of the space-charge layer and the amount of $\mathrm{BB}$ decreases. This is manifested in an increase of the transferred charge and a corresponding increase in the adsorption energy in Fig. 13. With $\Delta Q$ also $\Delta \Phi_{\text {surf }}$ increases, and the BB contribution $\Delta \Phi_{\mathrm{LR}}$ reduces accordingly. For heavily $n$-doped $\mathrm{ZnO}$, as found in transparent conductors, flat-band conditions are reached as band bending is now vanishingly small. In this regime, the adsorption energy of F4TCNQ has increased by more than $2 \mathrm{eV}$ to a value of $4.0 \mathrm{eV}$, as charge transfer is now almost one electron.

While the buildup of a space-charge layer is not unexpected, the magnitude of its effect is astounding. Our calculations show that it is now possible to include the bulk-doping dependence in first-principles calculations of surfaces and that its effects can be pronounced. The bulk doping concentration and the associated space-charge layer therefore provide an additional way to tune the interface properties in hybrid organic-inorganic systems. Bandbending engineering can now be used to tailor the energetic landscape at i/o interfaces, as recently demonstrated for ternary interfaces that use organic self-assembled monolayers to control the amount of band bending [134].

To summarize, we observed upward surface BB for F4TCNQ on the $\mathrm{ZnO}(000 \overline{1})$ surface, with a magnitude that is tunable by the bulk doping concentration of $\mathrm{ZnO}$. This behavior starkly contrasts with the downward surface $\mathrm{BB}$ induced by adsorption of atomic hydrogen on the $\mathrm{ZnO}(10 \overline{1} 0)$ surface discussed in Section 5. There, the donor-induced BB immediately led to a crossing of the Fermi energy and the CB minimum. The resulting partial filling of the CB at the surface can be viewed as "surface doping". As such, it is independent of the bulk doping concentration and will always 
lead to short-range BB in the order of a few $\AA$, which - for acceptors such as F4TCNQ - only occurs in the high doping regime. The penetration depth of surface BB, which depends on the Thomas-Fermi screening length, however, is crucial for the width of electron and hole injection barriers. An extended barrier, present for F4TCNQ acceptors on $\mathrm{ZnO}$ at low to intermediate doping concentrations, fully suppresses charge injection by electron tunnelling. The height of the barrier will then determine the injection efficiency. In contrast to this full suppression of tunnelling, narrow barriers - as found for hydrogen donors in Section 5 - permit tunnelling and thus facilitate hole injection on ultrafast timescales, even at energies of the bulk VB maximum.

\section{Summary and Conclusions}

The work function of a semiconductor forms the basis for the alignment of energy levels of any adsorbed species. In this Perspective, we have shown, by applying one- and two-photon photoemission and density-functional theory to the example of $\mathrm{ZnO}$, how sensitive the work function can be to the various adsorption-induced mechanisms: Long- and short-range charge transfer, changes of surface BB, bond dipole formation, as well as the permanent dipole moment of the adsorbed species. We discussed these mechanisms for the three most relevant cases of adsorption on $n$-type semiconductors, illustrated in Fig. 1: a) donor species, leading to downward surface BB, b) dipolar molecules, and c) acceptor species, resulting in upward surface BB. We found that both local and global effects affect the work function of the sample and that all need to be considered for accurate predictions of novel functional i/o interfaces.

In all three examples, we used natively $n$-type doped $\mathrm{ZnO}$ to illustrate the often forgotten truism that the doping type does not dictate the direction of $\mathrm{BB}$ and thus the creation of electron/hole injection barriers. The richness of phenomena and mechanisms we discovered in these three exemplary, well-defined systems demonstrates and confirms the potential of $\mathrm{ZnO}$ as a promising material for opto-electronic and light-harvesting applications, which may also involve other surface modifications [135]. Real i/o interfaces are, however, typically not as "ideal" as the ones discussed here. Realistic models would have to include the spatial profile of the dopants and information on other impurities at or near the interface (e.g., oxygen vacancies [31]) that could pin the Fermi level at defect levels [136] and limit the amount of BB. Nevertheless, mastering the notoriously challenging material $\mathrm{ZnO}$ in 
complex systems continues to be appealing from both the fundamental science and the technological perspectives.

\section{Acknowledgements}

We gratefully acknowledge funding through the Collaborative Research Center (Sfb) 951 of the German Science Foundation and are thankful for the significant contributions of all co-authors of Refs. $[9,8,10,11,12]$, in particular J.-C. Deinert and O. Hofmann for their experimental and theoretical contributions to this work, respectively. PR acknowledges support by the Academy of Finland through its Centres of Excellence Programme under project numbers 251748 and 284621. JS acknowledges funding from the European Commission project CRONOS (Grant No. 280879-2).

[1] C. Klingshirn, J. Fallert, H. Zhou, J. Sartor, C. Thiele, F. MaierFlaig, D. Schneider, H. Kalt, 65 years of $\mathrm{ZnO}$ research - old and very recent results, Physica Status Solidi (b) 247 (6) (2010) 1424. doi:10.1002/pssb.200983195.

[2] P. D. C. King, T. D. Veal, Conductivity in transparent oxide semiconductors, Journal of Physics: Condensed Matter 23 (33) (2011) 334214. doi:10.1088/0953-8984/23/33/334214.

[3] D. S. Ginley, H. Hosono, D. C. Paine (Eds.), Handbook of Transparent Conductors, Vol. 1, Springer US, 2011. doi:10.1007/978-1-4419-1638-9.

[4] C. J. Brabec, J. A. Hauch, P. Schilinsky, C. Waldauf, Production aspects of organic photovoltaics and their impact on the commercialization of devices, MRS Bulletin 30 (2005) 50. doi:10.1557/mrs2005.10.

[5] R. Schlesinger, F. Bianchi, S. Blumstengel, C. Christodoulou, R. Ovsyannikov, B. Kobin, K. Moudgil, S. Barlow, S. Hecht, S. R. Marder, F. Henneberger, N. Koch, Efficient light emission from inorganic and organic semiconductor hybrid structures by energy-level tuning, Nat Commun 6 (2015) 3044. doi:10.1038/ncomms7754.

[6] P. W. Bohn, Aspects of structure and energy transport in artificial molecular assemblies, Annu. Rev. Phys. Chem. 44 (1993) 37.

[7] D. Thomas, The exciton spectrum of zinc oxide, J. Phys. Chem. Solids 15 (1960) 86. 
[8] J.-C. Deinert, O. T. Hofmann, M. Meyer, P. Rinke, J. Stähler, Local aspects of hydrogen-induced metallization of the $\mathrm{ZnO}(10 \overline{1} 0)$ surface, Phys. Rev. B 91 (2015) 235313. doi:10.1103/PhysRevB.91.235313.

[9] J.-C. Deinert, D. Wegkamp, M. Meyer, C. Richter, M. Wolf, J. Stähler, Ultrafast exciton formation at the $\mathrm{ZnO}(10 \overline{1} 0)$ surface, Physical Review Letters 113 (5) (2014) 057602. doi:10.1103/PhysRevLett.113.057602.

[10] O. T. Hofmann, J.-C. Deinert, Y. Xu, P. Rinke, J. Stähler, M. Wolf, M. Scheffler, Large work function reduction by adsorption of a molecule with a negative electron affinity: Pyridine on $\mathrm{ZnO}(10 \overline{1} 0)$, J. Chem. Phys. 139 (2013) 174701.

[11] R. Schlesinger, Y. Xu, O. T. Hofmann, S. Winkler, J. Frisch, J. Niederhausen, A. Vollmer, S. Blumstengel, F. Henneberger, P. Rinke, M. Scheffler, N. Koch, Controlling the work function of $\mathrm{ZnO}$ and the energy-level alignment at the interface to organic semiconductors with a molecular electron acceptor, Phys. Rev. B 87 (2013) 155311.

[12] Y. Xu, O. T. Hofmann, R. Schlesinger, S. Winkler, J. Frisch, J. Niederhausen, A. Vollmer, S. Blumstengel, F. Henneberger, N. Koch, P. Rinke, M. Scheffler, Space-charge transfer in hybrid inorganicorganic systems, Phys. Rev. Lett. 111 (2013) 226802.

[13] H. Ishii, K. Sugiyama, E. Ito, K. Seki, Energy level alignment and interfacial electronic structures at organic/metal and organic/organic interfaces, Advanced Materials 11 (8) (1999) 605. doi:10.1002/(SICI)15214095(199906)11:8¡605::AID-ADMA605;3.0.CO;2-Q.

[14] X.-Y. Zhu, Electronic structure and electron dynamics at moleculemetal interfaces: implications for molecule-based electronics, Surface Science Reports 56 (12) (2004) 1 - 83. doi:http://dx.doi.org/10.1016/j.surfrep.2004.09.002.

[15] I. G. Hill, A. Rajagopal, A. Kahn, Y. Hu, Molecular level alignment at organic semiconductor-metal interfaces, Applied Physics Letters 73 (5) (1998) 662-664. doi:http://dx.doi.org/10.1063/1.121940.

[16] D. Cahen, A. Kahn, Electron energetics at surfaces and interfaces: Concepts and experiments, Advanced Materials 15 (4) (2003) 271-277. 
doi:10.1002/adma.200390065.

URL http://dx.doi.org/10.1002/adma. 200390065

[17] A. Klein, C. Krber, A. Wachau, F. Suberlich, Y. Gassenbauer, S. P. Harvey, D. E. Proffit, T. O. Mason, Transparent conducting oxides for photovoltaics: Manipulation of fermi level, work function and energy band alignment, Materials 3 (11) (2010) 4892. doi:10.3390/ma3114892.

[18] B. Gumhalter, H. Petek, Decoherence effects in propagation of optically generated electronhole pairs in image potential states, Surface Science 445 (23) (2000) 195 - 208. doi:http://dx.doi.org/10.1016/S00396028(99)01037-7.

[19] E. Chulkov, V. Silkin, P. Echenique, Image potential states on lithium, copper and silver surfaces, Surface Science 391 (1) (1997) L1217L1223. doi:http://dx.doi.org/10.1016/S0039-6028(97)00653-5.

URL http://www. sciencedirect.com/science/article/pii/S0039602897006535

[20] U. Höfer, I. L. Shumay, C. Reuß, U. Thomann, W. Wallauer, T. Fauster, Time-resolved coherent photoelectron spectroscopy of quantized electronic states on metal surfaces, Science 277 (5331) (1997) 1480-1482. doi:10.1126/science.277.5331.1480.

[21] M. Wolf, E. Knoesel, T. Hertel, Ultrafast dynamics of electrons in image-potential states on clean and xe-covered cu(111), Phys. Rev. B 54 (1996) R5295-R5298. doi:10.1103/PhysRevB.54.R5295.

[22] S. Schuppler, N. Fischer, T. Fauster, W. Steinmann, Lifetime of imagepotential states on metal surfaces, Phys. Rev. B 46 (1992) 13539-13547. doi:10.1103/PhysRevB.46.13539.

[23] Z. Zhang, J. T. J. Yates, Band bending in semiconductors: Chemical and physical consequences at surfaces and interfaces, Chemical Reviews 112 (2012) 5520 .

[24] A. Argondizzo, X. Cui, C. Wang, H. Sun, H. Shang, J. Zhao, H. Petek, Ultrafast multiphoton pump-probe photoemission excitation pathways in rutile $\mathrm{TiO}_{2}(110)$, Phys. Rev. B 91 (2015) 155429. doi:10.1103/PhysRevB.91.155429. 
[25] A. Argondizzo, S. Tan, H. Petek, Resonant two-photon photoemission from Ti 3d defect states of $\mathrm{TiO}_{2}(110)$ revisited, J. Phys. Chem. C 120 (2016) 12959. doi:10.1021/acs.jpcc.6b04517.

[26] Z. Wang, B. Wen, Q. Hao, L.-M. Liu, C. Zhou, X. Mao, X. Lang, W.-J. Yin, D. Dai, A. Selloni, X. Yang, Localized excitation of $\mathrm{Ti}^{3+}$ ions in the photoabsorption and photocatalytic activity of reduced rutile $\mathrm{TiO}_{2}(110)$, J. Am. Chem. Soc. 137 (2015) 9146. doi:10.1021/jacs.5b04483.

[27] Y. Zhang, D. T. Payne, C. L. Pang, H. H. Fielding, G. Thornton, Nonband-gap photoexcitation of hydroxylated $\mathrm{TiO}_{2}(110)$, J. Phys. Chem. Lett. 6 (2015) 3391. doi:10.1021/acs.jpclett.5b01508.

[28] C. Klingshirn, ZnO: From basics towards applications, Physica Status Solidi (b) 244 (9) (2007) 3027. doi:10.1002/pssb.200743072.

[29] B. Meyer, D. Marx, Density-functional study of the structure and stability of ZnO surfaces, Physical Review B 67 (3) (2003) 035403. doi:10.1103/PhysRevB.67.035403.

[30] V. Staemmler, K. Fink, B. Meyer, D. Marx, M. Kunat, S. Gil Girol, U. Burghaus, C. Wöll, Stabilization of polar ZnO surfaces: Validating microscopic models by using $\mathrm{CO}$ as a probe molecule, Physical Review Letters 90 (10) (2003) 106102. doi:10.1103/PhysRevLett.90.106102.

[31] U. Özgür, Y. I. Alivov, C. Liu, A. Teke, M. A. Reshchikov, S. Dogan, V. Avrutin, S.-J. Cho, H. Morkoc, A comprehensive review of $\mathrm{ZnO}$ materials and devices, J. Appl. Phys. 98 (4) (2005) 041301.

[32] C. Wöll, The chemistry and physics of zinc oxide surfaces, Progress in Surface Science 82 (2007) 55. doi:dx.doi.org/10.1016/j.progsurf.2006.12.002.

[33] X.-L. Yin, A. Birkner, K. Hänel, T. Löber, U. Köhler, C. Wöll, Adsorption of atomic hydrogen on $\mathrm{ZnO}(10 \overline{1} 0)$ : STM study, Phys. Chem. Chem. Phys. 8 (13) (2006) 1477. doi:10.1039/b515464b.

[34] M. Valtiner, M. Todorova, G. Grundmeier, J. Neugebauer, Temperature stabilized surface reconstructions at polar $\mathrm{ZnO}(0001)$, Phys. Rev. Lett. 103 (2009) 065502. doi:10.1103/PhysRevLett.103.065502. 
[35] M. Valtiner, M. Todorova, J. Neugebauer, Hydrogen adsorption on polar $\mathrm{ZnO}(0001)-\mathrm{Zn}$ : Extending equilibrium surface phase diagrams to kinetically stabilized structures, Phys. Rev. B 82 (2010) 165418. doi:10.1103/PhysRevB.82.165418.

[36] J. V. Lauritsen, S. Porsgaard, M. K. Rasmussen, M. C. R. Jensen, R. Bechstein, K. Meinander, B. S. Clausen, S. Helveg, R. Wahl, G. Kresse, F. Besenbacher, Stabilization principles for polar surfaces of ZnO, ACS Nano 5 (7) (2011) 5987-5994. doi:10.1021/nn2017606.

[37] R. Wahl, J. V. Lauritsen, F. Besenbacher, G. Kresse, Stabilization mechanism for the polar $\mathrm{ZnO}(000 \overline{1})-\mathrm{O}$ surface, Phys. Rev. B 87 (2013) 085313. doi:10.1103/PhysRevB.87.085313.

[38] H. Sezen, H. Shang, F. Bebensee, C. Yang, M. Buchholz, A. Nefedov, S. Heissler, C. Carbogno, M. Scheffler, P. Rinke, C. Wöll, Evidence for photogenerated intermediate hole polarons in $\mathrm{ZnO}$, Nat. Commun. 6 (2015) 6901.

[39] H. Sezen, H. Shang, F. Bebensee, C. Yang, M. Buchholz, A. Nefedov, S. Heissler, C. Carbogno, M. Scheffler, P. Rinke, C. Wöll, Corrigendum: Evidence for photogenerated intermediate hole polarons in $\mathrm{ZnO}$, Nat. Commun 6 (2015) 7694.

[40] P. D. C. King, T. D. Ginley, C. F. McConville, J. Zúñiga Pérez, V. Muñoz Sanjosé, M. Hopkinson, E. D. L. Rienks, M. F. Jensen, P. Hofmann, Surface band-gap narrowing in quantized electron accumulation layers, Physical Review Letters 104 (25) (2010) 256803. doi:10.1103/PhysRevLett.104.256803.

[41] A. Janotti, C. G. Van de Walle, Fundamentals of zinc oxide as a semiconductor, Reports on Progress in Physics 72 (12) (2009) 126501. doi:10.1088/0034-4885/72/12/126501.

[42] K. Fink, Ab initio cluster calculations on the electronic structure of oxygen vacancies at the polar $\mathrm{ZnO}(000 \overline{1})$ surface and on the adsorption of $\mathrm{H}_{2}, \mathrm{CO}$, and $\mathrm{CO}_{2}$ at these sites., Physical Chemistry Chemical Physics 8 (13) (2006) 1482. doi:10.1039/b515907e. 
[43] H. T. Wang, B. S. Kang, F. Ren, L. C. Tien, P. W. Sadik, D. P. Norton, S. J. Pearton, J. Lin, Hydrogen-selective sensing at room temperature with ZnO nanorod, Applied Physics Letters 86 (24) (2005) 243503.

[44] M. Timpel, M. Nardi, S. Krause, G. Ligorio, C. Christodoulou, L. Pasquali, A. Giglia, J. Frisch, B. Wegner, P. Moras, N. Koch, Surface modification of $\mathrm{ZnO}(0001) \mathrm{Zn}$ with phosphonate-based self- assembled monolayers: Binding modes, orientation, and work function, Chem. Mater. 26 (2014) 5042.

[45] I. Lange, S. Reiter, M. Pätzel, A. Zykov, A. Nefedov, J. Hildebrandt, S. Hecht, S. Kowarik, C. Wöll, G. Heimel, D. Neher, Tuning the work function of polar zinc oxide surfaces using modified phosphonic acid self-assembled monolayers, Adv. Funct. Mater. 24 (2014) 7014.

[46] K. Sundaram, A. Khan, Work function determination of zinc oxide films, J. Vac. Sci. Technol. A 15 (1997) 428.

[47] W. Göpel, L. Brillson, C. Brucker, Surface point defects and schottky barrier formation on $\mathrm{ZnO}(10 \overline{1} 0)$, J. Vac. Sci. Technol. 17 (1980) 894.

[48] K. Jacobi, G. Zwicker, A. Gutmann, Work function, electron affinity and band bending of zinc oxide surfaces, Surface Science 141 (1) (1984) 109. doi:10.1016/0039-6028(84)90199-7.

[49] H. Moormann, D. Kohl, G. Heiland, Variations of work function and surface conductivity on clean cleaved zinc oxide surfaces by annealing and by hydrogen adsorption, Surface Science 100 (2) (1980) 302 - 314. doi:10.1016/0039-6028(80)90374-X.

[50] W. A. Tisdale, M. Muntwiler, D. J. Norris, E. S. Aydil, X.-Y. Zhu, Electron dynamics at the $\mathrm{ZnO}(10 \overline{1} 0)$ surface, The Journal of Physical Chemistry C 112 (37) (2008) 14682. doi:10.1021/jp802455p.

[51] H. Lüth, Solid Surfaces, Interfaces and Thin Films, 5th Edition, Graduate Texts in Physics, Springer Berlin Heidelberg, 2010. doi:10.1007/978-3-642-13592-7.

[52] K. Ozawa, K. Mase, Metallization of $\mathrm{ZnO}(10 \overline{1} 0)$ by adsorption of hydrogen, methanol, and water: Angle-resolved photoelectron spectroscopy, Phys. Rev. B 81 (20) (2010) 205322. doi:10.1103/PhysRevB.81.205322. 
[53] K. Ozawa, K. Mase, Comparison of the surface electronic structures of $\mathrm{H}$ adsorbed $\mathrm{ZnO}$ surfaces: An angle-resolved photoelectron spectroscopy study, Phys. Rev. B 83 (12) (2011) 125406. doi:10.1103/PhysRevB.83.125406.

[54] Y. Wang, B. Meyer, X. Yin, M. Kunat, D. Langenberg, F. Traeger, A. Birkner, C. Wöll, Hydrogen induced metallicity on the $\mathrm{ZnO}(10 \overline{1} 0)$ surface, Phys. Rev. Lett. 95 (2005) 266104. doi:10.1103/PhysRevLett.95.266104.

[55] A. Tkatchenko, M. Scheffler, Accurate molecular van der Waals interactions from ground-state electron density and free-atom reference data, Phys. Rev. Lett. 102 (2009) 073005.

[56] V. Ruiz, W. Liu, E. Zojer, M. Scheffler, A. Tkatchenko, Densityfunctional theory with screened van der Waals interactions for the modeling of hybrid inorganic-organic systems, Physical Review Letters 108 (14) (2012) 146103. doi:10.1103/PhysRevLett.108.146103.

[57] J. Neaton, M. Hybertsen, S. Louie, Renormalization of molecular electronic levels at metal-molecule interfaces, Physical Review Letters 97 (21). doi:10.1103/PhysRevLett.97.216405.

[58] C. Freysoldt, P. Rinke, M. Scheffler, Controlling polarization at insulating surfaces: Quasiparticle calculations for molecules adsorbed on insulator films, Phys. Rev. Lett. 103 (2009) 056803.

[59] K. Thygesen, A. Rubio, Renormalization of molecular quasiparticle levels at metal-molecule interfaces: Trends across binding regimes, Physical Review Letters 102 (4). doi:10.1103/PhysRevLett.102.046802.

[60] J. M. Garcia-Lastra, K. S. Thygesen, Renormalization of optical excitations in molecules near a metal surface, Phys. Rev. Lett. 106 (2011) 187402. doi:10.1103/PhysRevLett.106.187402.

URL http://link.aps.org/doi/10.1103/PhysRevLett.106.187402

[61] A. Biller, I. Tamblyn, J. B. Neaton, L. Kronik, Electronic level alignment at a metal-molecule interface from a short-range hybrid functional, The Journal of Chemical Physics 135 (16) (2011) 164706. doi:10.1063/1.3655357. 
[62] J. P. Perdew, A. Zunger, Self-interaction correction to densityfunctional approximations for many-electron systems, Phys. Rev. B 23 (1981) 5048.

[63] J. P. Perdew, R. G. Parr, M. Levy, J. L. Balduz, Density-functional theory for fractional particle number: Derivative discontinuities of the energy, Phys. Rev. Lett. 49 (1982) 1691.

[64] O. T. Hofmann, V. Atalla, N. Moll, P. Rinke, M. Scheffler, Interface dipoles of organic molecules on $\mathrm{Ag}(111)$ in hybrid density-functional theory, New J. Phys. 15 (12) (2013) 123028.

[65] F. Caruso, V. Atalla, X. Ren, A. Rubio, M. Scheffler, P. Rinke, Firstprinciples description of charge transfer in donor-acceptor compounds from self-consistent many-body perturbation theory, Phys. Rev. B 90 (2014) 085141.

[66] O. T. Hofmann, P. Rinke, M. Scheffler, G. Heimel, Integer versus fractional charge transfer at metal(/insulator)/organic interfaces: $\mathrm{Cu}(/ \mathrm{NaCl}) / \mathrm{TCNE}, \mathrm{ACS}$ Nano 9 (5) (2015) 5391.

[67] O. T. Hofmann, P. Rinke, M. Scheffler, G. Heimel, Correction to integer versus fractional charge transfer at metal(/insulator)/organic interfaces: $\mathrm{Cu}(/ \mathrm{NaCl}) / \mathrm{TCNE}$, ACS Nano 9 (8) (2015) 8637.

[68] J. I. Martínez, F. Flores, J. Ortega, S. Rangan, C. Ruggieri, R. Bartynski, Chemical interaction, space-charge layer, and molecule charging energy for a $\mathrm{TiO}_{2} / \mathrm{TCNQ}$ interface, The Journal of Physical Chemistry C 119 (38) (2015) 22086.

[69] S. Rangan, C. Ruggieri, R. Bartynski, J. I. Martınez, F. Flores, J. Ortega, Densely packed ZnTPPs monolayer on the rutile $\mathrm{TiO}_{2}(110)-(1$ $\mathrm{x}$ 1) surface: Adsorption behavior and energy level alignment, The Journal of Physical Chemistry C 120 (8) (2016) 4430.

[70] N. A. Richter, S. Sicolo, S. V. Levchenko, J. Sauer, M. Scheffler, Concentration of vacancies at metal-oxide surfaces: Case study of MgO(100), Phys. Rev. Lett. 111 (2013) 045502. 
[71] O. Sinai, O. T. Hofmann, P. Rinke, M. Scheffler, G. Heimel, L. Kronik, Multiscale approach to the electronic structure of doped semiconductor surfaces, Phys. Rev. B 91 (2015) 075311.

[72] R. Fischer, S. Schuppler, N. Fischer, T. Fauster, W. Steinmann, Image states and local work function for ag/pd(111), Phys. Rev. Lett. 70 (1993) 654. doi:10.1103/PhysRevLett.70.654.

[73] D. Niesner, T. Fauster, J. I. Dadap, N. Zaki, K. R. Knox, P.-C. Yeh, R. Bhandari, R. M. Osgood, M. Petrović, M. Kralj, Trapping surface electrons on graphene layers and islands, Phys. Rev. B 85 (2012) 081402. doi:10.1103/PhysRevB.85.081402.

[74] D. Wegkamp, M. Meyer, C. Richter, M. Wolf, J. Stähler, Photoinduced work function modifications and their effect on photoelectron spectroscopy, Appl. Phys. Lett. 103 (15) (2013) 151603. doi:10.1063/1.4823591.

[75] J. Neugebauer, M. Scheffler, Adsorbate-substrate and adsorbateadsorbate interactions of $\mathrm{Na}$ and $\mathrm{K}$ adlayers on $\mathrm{Al}(111)$, Physical Review B 46 (24) (1992) 16067. doi:10.1103/PhysRevB.46.16067.

[76] K. Onda, B. Li, H. Petek, Two-photon photoemission spectroscopy of $\mathrm{Tio}_{2}(110)$ surfaces modified by defects and $\mathrm{o}_{2}$ or $\mathrm{h}_{2} \mathrm{O}$ adsorbates, Phys. Rev. B 70 (2004) 045415. doi:10.1103/PhysRevB.70.045415. URL http://link.aps.org/doi/10.1103/PhysRevB.70.045415

[77] N. Sai, K. Leung, J. Chelikowsky, Hybrid density functional study of oligothiophene/ZnO interface for photovoltaics, Physical Review B 83 (12) (2011) 121309(R).

[78] H. Li, L. K. Schirra, J. Shim, H. Cheun, B. Kippelen, O. L. A. Monti, J.-L. Bredas, Zinc oxide as a model transparent conducting oxide: A theoretical and experimental study of the impact of hydroxylation, vacancies, interstitials, and extrinsic doping on the electronic properties of the polar $\mathrm{ZnO}$ (0002) surface, Chemistry of Materials 24 (15) (2012) 3044 .

[79] K. Noori, F. Giustino, Ideal energy-level alignment at the ZnO/P3HT photovoltaic interface, Advanced Functional Materials 22 (24) (2012) 5089-5095. 
[80] N. Moll, Y. Xu, O. T. Hofmann, P. Rinke, Stabilization of semiconductor surfaces through bulk dopants, New J. Phys. 15 (8) (2013) 083009.

[81] P. Winget, L. K. Schirra, D. Cornil, H. Li, V. Coropceanu, P. F. Ndione, A. K. Sigdel, D. S. Ginley, J. J. Berry, J. Shim, H. Kim, B. Kippelen, J.-L. Brédas, O. L. A. Monti, Defect-driven interfacial electronic structures at an organic/metal-oxide semiconductor heterojunction, Advanced Materials 26 (27) (2014) 4711.

[82] G. Wu, Z. Li, X. Zhang, G. Lu, Charge separation and exciton dynamics at polymer/ZnO interface from first-principles simulations, The Journal of Physical Chemistry Letters 5 (15) (2014) 2649.

[83] B. Bieniek, O. T. Hofmann, P. Rinke, Influence of hydrogen on the structure and stability of ultra-thin $\mathrm{ZnO}$ on metal substrates, Applied Physics Letters 106 (13) (2015) 131602.

[84] M. Gruenewald, L. K. Schirra, P. Winget, M. Kozlik, P. F. Ndione, A. K. Sigdel, J. J. Berry, R. Forker, J.-L. Brédas, T. Fritz, O. L. A. Monti, Integer charge transfer and hybridization at an organic semiconductor/conductive oxide interface, The Journal of Physical Chemistry C 119 (9) (2015) 4865.

[85] J.-P. Niemela, A. J. Karttunen, M. Karppinen, Inorganic-organic superlattice thin films for thermoelectrics, J. Mater. Chem. C 3 (2015) 10349.

[86] A. J. Karttunen, T. Tynell, M. Karppinen, Atomic-level structural and electronic properties of hybrid inorganic-organic ZnO:hydroquinone superlattices fabricated by ALD/MLD, The Journal of Physical Chemistry C 119 (23) (2015) 13105.

[87] A. J. Karttunen, T. Tynell, M. Karppinen, Layer-by-layer design of nanostructured thermoelectrics: First-principles study of ZnO:organic superlattices fabricated by ALD/MLD, Nano Energy 22 (2016) 338.

[88] L. Hedin, Phys. Rev. 139 (1965) A796.

[89] F. Aryasetiawan, O. Gunnarsson, The gw method, Rep. Prog. Phys. 61 (1998) 237. 
[90] L. Hedin, On correlation effects in electron spectroscopies and the $g w$ approximation, J. Phys.: Condens. Matter 11 (1999) R489.

[91] W. G. Aulbur, L. Jönsson, J. W. Wilkins, Quasiparticle calculations in solids, Solid State Phys. 54 (2000) 1.

[92] G. Onida, L. Reining, A. Rubio, Electronic excitations: densityfunctional versus many-body green's function approaches, Rev. Mod. Phys. 74 (2002) 601.

[93] P. Rinke, A. Qteish, J. Neugebauer, C. Freysoldt, M. Scheffler, Combining $G W$ calculations with exact-exchange density-functional theory: An analysis of valence-band photoemission for compound semiconductors, New J. Phys. 7 (2005) 126.

[94] M. Giantomassi, M. Stankovski, R. Shaltaf, M. Grüning, F. Bruneval, P. Rinke, G.-M. Rignanese, Electronic properties of interfaces and defects from many-body perturbation theory: Recent developments and applications, physica status solidi (b) 248 (2) (2011) 275-289.

[95] C. Freysoldt, P. Eggert, P. Rinke, A. Schindlmayr, M. Scheffler, Screening in 2d: $G W$ calculations for surfaces and thin films using the repeated-slab approach, Phys. Rev. B 77 (2008) 235428.

[96] M. Rohlfing, N.-P. Wang, P. Krüger, J. Pollmann, Image states and excitons at insulator surfaces with negative electron affinity, Phys. Rev. Lett. 91 (2003) 256802.

[97] M. Kutschera, M. Weinelt, M. Rohlfing, T. Fauster, Image-potentialinduced surface state at si(100), Applied Physics A 88 (3) (2007) 519526. doi:10.1007/s00339-007-4074-x.

[98] A. Greuling, M. Rohlfing, R. Temirov, F. S. Tautz, F. B. Anders, $A b$ initio study of a mechanically gated molecule: From weak to strong correlation, Phys. Rev. B 84 (2011) 125413. doi:10.1103/PhysRevB.84.125413.

URL http://link.aps.org/doi/10.1103/PhysRevB.84.125413

[99] C. E. Patrick, F. Giustino, Quantitative analysis of valence photoemission spectra and quasiparticle excitations at chromophoresemiconductor interfaces, Phys. Rev. Lett. 109 (2012) 116801. doi:10.1103/PhysRevLett.109.116801. 
[100] P. Umari, L. Giacomazzi, F. D. Angelis, M. Pastore, S. Baroni, Energy-level alignment in organic dye-sensitized tio ${ }_{2}$ from gw calculations, The Journal of Chemical Physics 139 (1) (2013) 014709. doi:10.1063/1.4809994.

[101] M. Yu, P. Doak, I. Tamblyn, J. B. Neaton, Theory of covalent adsorbate frontier orbital energies on functionalized light-absorbing semiconductor surfaces, J. Phys. Chem. Lett. 4 (10) (2013) 1701-1706.

[102] A. Migani, D. J. Mowbray, A. Iacomino, J. Zhao, H. Petek, A. Rubio, Level alignment of a prototypical photocatalytic system: Methanol on tio2(110), Journal of the American Chemical Society 135 (31) (2013) 11429-11432. doi:10.1021/ja4036994.

[103] L.-H. Li, O. Y. Kontsevoi, A. J. Freeman, Orientation-dependent electronic structures and optical properties of the p3ht:pcbm interface: A first-principles gw-bse study, The Journal of Physical Chemistry C 118 (19) (2014) 10263-10270.

[104] A. Migani, D. J. Mowbray, Coverage dependence of the level alignment for methanol on tio2, Computational and Theoretical Chemistry 1040 - 1041 (0) (2014) 259 - 265, excited states: From isolated molecules to complex environments Excited states.

[105] A. Migani, D. J. Mowbray, J. Zhao, H. Petek, A. Rubio, Quasiparticle level alignment for photocatalytic interfaces, Journal of Chemical Theory and Computation 10 (5) (2014) 2103-2113.

[106] C. Verdi, E. Mosconi, F. De Angelis, M. Marsili, P. Umari, Alignment of energy levels in dye/semiconductor interfaces by $g w$ calculations: Effects due to coadsorption of solvent molecules, Phys. Rev. B 90 (2014) 155410 .

[107] M. Alves-Santos, L. M. M. Jorge, M. J. Caldas, D. Varsano, Electronic structure of interfaces between thiophene and tio2 nanostructures, The Journal of Physical Chemistry C 118 (25) (2014) 1353913544. doi:10.1021/jp407275e.

[108] A. Migani, D. J. Mowbray, J. Zhao, H. Petek, Quasiparticle interfacial level alignment of highly hybridized frontier levels: H2o on tio2(110), Journal of Chemical Theory and Computation 11 (1) (2015) 239-251. 
[109] D. J. Mowbray, A. Migani, Using g0w0 level alignment to identify catechol's structure on tio2(110), The Journal of Physical Chemistry C 119 (34) (2015) 19634-19641.

[110] J. Ihm, A. Zunger, M. L. Cohen, Momentum-space formalism for the total energy of solids, J. Phys. C 12 (21) (1979) 4409.

[111] H.-P. Komsa, A. Pasquarello, Finite-size supercell correction for charged defects at surfaces and interfaces, Phys. Rev. Lett. 110 (9) (2013) 095505.

[112] D. Wang, D. Han, X.-B. Li, S.-Y. Xie, N.-K. Chen, W. Q. Tian, D. West, H.-B. Sun, S. B. Zhang, Determination of formation and ionization energies of charged defects in two-dimensional materials, Phys. Rev. Lett. 114 (2015) 196801.

[113] O. Sinai, L. Kronik, Simulated doping of Si from first principles using pseudoatoms, Phys. Rev. B 87 (23) (2013) 235305.

[114] S. M. Sze, K. K. Ng, Physics of Semiconductor Devices, WileyInterscience, Hoboken, New Jersey, 2006.

[115] V. Blum, R. Gehrke, F. Hanke, P. Havu, V. Havu, X. Ren, K. Reuter, M. Scheffler, Ab initio molecular simulations with numeric atomcentered orbitals, Computer Physics Communications 180 (11) (2009) 2175 .

[116] V. Havu, V. Blum, P. Havu, M. Scheffler, Efficient integration for allelectron electronic structure calculation using numeric basis functions, Journal of Computational Physics 228 (22) (2009) 8367.

[117] X. Ren, P. Rinke, V. Blum, J. Wieferink, A. Tkatchenko, S. Andrea, K. Reuter, V. Blum, M. Scheffler, Resolution-of-identity approach to Hartree-Fock, hybrid density functionals, RPA, MP2, and GW with numeric atom-centered orbital basis functions, New J. Phys. 14 (2012) 053020 .

[118] S. V. Levchenko, X. Ren, J. Wieferink, R. Johanni, P. Rinke, V. Blum, M. Scheffler, Hybrid functionals for large periodic systems in an allelectron, numeric atom-centered basis framework, Computer Physics Communications 192 (2015) 60. 
[119] J. P. Perdew, K. Burke, M. Ernzerhof, Generalized gradient approximation made simple, Physical Review Letters 77 (18) (1996) 3865.

[120] J. Heyd, G. E. Scuseria, M. Ernzerhof, Hybrid functionals based on a screened coulomb potential, The Journal of Chemical Physics 118 (18) (2003) 8207. doi:10.1063/1.1564060.

[121] A. V. Krukau, O. A. Vydrov, A. F. Izmaylov, G. E. Scuseria, Influence of the exchange screening parameter on the performance of screened hybrid functionals, The Journal of Chemical Physics 125 (22) (2006) 224106.

[122] G.-X. Zhang, A. Tkatchenko, J. Paier, H. Appel, M. Scheffler, van der Waals interactions in ionic and semiconductor solids, Physical Review Letters 107 (24) (2011) 245501.

[123] V. P. Zhukov, P. M. Echenique, E. V. Chulkov, Two types of excited electron dynamics in zinc oxide, Phys. Rev. B 82 (2010) 094302. doi:10.1103/PhysRevB.82.094302.

[124] J.-C. Deinert, Zinc oxide surfaces and interfaces: Electronic structure and dynamics of excited states, Ph.D. thesis, Technische Universität Berlin (2015).

[125] A. Walsh, J. L. F. Da Silva, S.-H. Wei, Origins of band-gap renormalization in degenerately doped semiconductors, Phys. Rev. B 78 (2008) 075211. doi:10.1103/PhysRevB.78.075211.

[126] S. Hövel, C. Kolczewski, M. Wühn, J. Albers, K. Weiss, V. Staemmler, C. Wöll, Pyridine adsorption on the polar $\mathrm{ZnO(0001)} \mathrm{surface:} \mathrm{Zn}$ termination versus O termination, J. Chem. Phys. 112 (8) (2000) 3909. doi:10.1063/1.480942.

[127] J. Walsh, R. Davis, C. Muryn, G. Thornton, V. Dhanak, K. Prince, Orientation of benzene and pyridine on $\mathrm{ZnO}(10 \overline{1} 0)$, Physical Review B 48 (19) (1993) 14749.

[128] T. Fauster, H. Petek, M. Wolf, Dynamics at Solid State Surfaces and Interfaces, Vol. 2: Fundamentals, Wiley-Blackwell, 2012, Ch. Surface States and Adsorbate-Induced Electronic Structure, p. 115. 
[129] J. Zhao, N. Pontius, A. Winkelmann, V. Sametoglu, A. Kubo, A. G. Borisov, D. Sánchez-Portal, V. M. Silkin, E. V. Chulkov, P. M. Echenique, H. Petek, Electronic potential of a chemisorption interface, Phys. Rev. B 78 (2008) 085419. doi:10.1103/PhysRevB.78.085419.

[130] D. Heskett, T. Maeda Wong, A. Smith, W. Graham, N. DiNardo, E. W. Plummer, Correlation of alkali metal-induced work function changes on semiconductor and metal surfaces, J. Vac. Sci. Technol. B 7 (1989) 915.

[131] G. Heimel, L. Romaner, E. Zojer, J.-L. Brédas, Surface point defects and Schottky barrier formation on $\mathrm{ZnO}(10 \overline{1} 0)$, J. Vac. Sci. Technol. 17 (1980) 894.

[132] B. Meyer, First-principles study of the polar O-terminated ZnO surface in thermodynamic equilibrium with oxygen and hydrogen, Phys. Rev. B 69 (4) (2004) 045416. doi:10.1103/PhysRevB.69.045416.

[133] W. Mönch, Semiconductor Surfaces and Interfaces, Springer Series in Surface Science, Vol. 26, Springer, Berlin/Heidelberg/New York, 2001.

[134] O. T. Hofmann, P. Rinke, Band bending engineering at organic/inorganic interfaces using organic self-assembled monolayers, Advanced Electronic Materials.

[135] A. Ostapenko, T. Klffel, J. Euner, K. Harms, S. Dehnen, B. Meyer, G. Witte, Etching of crystalline zno surfaces upon phosphonic acid adsorption: Guidelines for the realization of well-engineered functional self-assembled monolayers, ACS Applied Materials \& Interfaces 8 (21) (2016) 13472-13483. doi:10.1021/acsami.6b02190.

[136] H. Mosbacker, Y. Strzhemechny, B. White, P. Smith, D. C. Look, D. Reynolds, C. Litton, L. Brillson, Role of near-surface states in Ohmic-Schottky conversion of $\mathrm{Au}$ contacts to $\mathrm{ZnO}$, Appl. Phys. Lett. 87 (1) (2005) 012102. 